\title{
Bildungen von „Parteiungen“( (dang) in der konfuzianischen Bürokratie im vormodernen China
}

\author{
Christian Meyer (D)
}

Eingegangen: 30. Januar 2021 / Überarbeitet: 6. November 2021 / Angenommen: 11. November 2021

(C) Der/die Autor(en) 2022

Zusammenfassung Der Artikel thematisiert die Frage von Parteiungen im vormodernen China im Spannungsfeld der bürokratischen Mechanismen einerseits und des Konfuzianismus als Leitideologie des kaiserlichen China andererseits. Er verfolgt zunächst begriffsgeschichtlich den Terminus dang 黨, der heute in Ostasien als Übersetzungsbegriff für ,Partei ‘ fungiert, jedoch im traditionellen China das Stichwort zu Debatten um Gruppenbildungen darstellte. Dieser war zumeist negativ als „Cliquenbildung“ zu Karrierezwecken konnotiert.

Am Beispiel der Gruppenbildungsstrukturen des 11. Jahrhunderts wird herausgearbeitet, von welchen Faktoren die Bildung von Parteiungen abhing. Der Artikel argumentiert, dass Parteiungen einerseits eine strukturelle Notwendigkeit der Gruppe- und Meinungsbildung innerhalb eines größeren kommunikativen Kontexts, der Bürokratie, darstellten, andererseits solche Gruppenbildung eng mit ideologischen Aspekten verbunden waren. Dies lässt sich anhand des Einflusses von innerkonfuzianischen intellektuellen Bewegungen wie der Guwen- und der neokonfuzianischen Daoxue-Bewegung ablesen. Parteiungsbildungen und intellektuelle Bewegungen korrelieren hier deutlich. Die kaiserlichen Opferriten hatten als symbolische Materie einen hohen Stellenwert in Parteiungskämpfen, Ritenfragen konnten als spezifische Konfliktlinien jedoch nur seltener oder nur zeitweise gruppenbildend wirken.

Die komplexe Dynamik kontinuierlicher Neuformierungen von ,Parteiungen“ (dang) in der Nördlichen Song-Dynastie (960-1126 n.Chr.) lässt sich insgesamt nur durch ein Zusammenwirken von Karriereinteressen, Loyalitätstrukturen und gruppenbildenden Ideologien entlang wechselnder Konfliktlinien innerhalb der Zivilbürokratie und der diese tragenden konfuzianischen Gelehrtenschicht analysieren. Sie spiegelt dabei das komplexe Ineinander von Konfuzianismus und Staat.

Christian Meyer $(\bowtie)$

Sinologie, Freie Universität Berlin, Berlin, Deutschland

E-Mail: ch.meyer@fu-berlin.de 
Der Begriff dang kann als chinesischer Keyterm dienen, der nicht nur eine komparative Perspektive auf das Thema ermöglicht, sondern durch seine lokale Begriffsgeschichte einen religionswissenschaftlichen Keyterm ,Parteiung ' (oder Englisch ,faction') mit Beobachtungen zu spezifischen außerwestlichen (chinesisch-konfuzianischen) Konstellationen bereichert.

Schlüsselwörter Parteiung $\cdot$ dang $\cdot$ pengdang $\cdot$ China $\cdot$ Konfuzianismus $\cdot$ Ouyang Xiu

\section{Formations of "Factions" (dang) in the Confucian bureaucracy in premodern China}

Abstract This article addresses the question of factions in pre-modern China between the poles of bureaucratic mechanisms on the one hand and Confucianism as a hegemonic ideology of Imperial China on the other hand. In a first step it pursues the conceptual history of the term dang 黨, that functions in East Asia nowadays as a translational term for 'party'. In traditional China, however, it is the keyword for debates on the formation of factional groups. It was used mainly in a negative way in the meaning of 'cliques' or 'networks' for the purpose of careers.

Using the example of group formations in the 11th century, the article analyzes which factors were relevant in the formation of factions. It argues that factions were on the one hand structurally necessary for group and opinion formation within a larger communicative context, such as a bureaucracy, however, on the other hand such group formations could often be identified by their ideological character and go clearly beyond purely political interests. This can be demonstrated by the examples of inner-Confucian movements such as the guwen and the daoxue movements. In these cases, the formation of political parties and intellectual movements correlate rather clearly. Imperial sacrificial rites had a high status as symbolic material in party struggles, but as a specific line of conflict such ritual questions would only rarely or rather temporarily lead to group formations.

The complex dynamics of continuous restructuring of 'factions' (dang) in the Northern Song dynasty (960-1126 AD) can therefore only be explained by the interplay of career interests, loyalties and group-forming ideologies along changing lines of conflict within the civil bureaucracy and the social group of Confucianeducated scholars. It reflects hereby the complex interpenetration of Confucianism and state.

The term dang can serve as Chinese key term that enables a comparative perspective on the topic. Furthermore, its local conceptual history can also enrich a key term 'faction' in the study of religions with observations on specific non-Western (Chinese) constellations.

Keywords Faction $\cdot$ dang $\cdot$ pengdang $\cdot$ China $\cdot$ Confucianism $\cdot$ Ouyang Xiu 


\section{Einleitung}

Ein Begriff Parteiung oder faction erscheint als ein Begriff der modernen westlichen Begriffsgeschichte. Soll er seine Eignung als Keyterm auch im vormodernen und vor allem auch im außerwestlichen Kontext erweisen, muss seine Anwendbarkeit auch in solchen Kontexten erprobt werden. Dies gilt auch und gerade für das Verhältnis von Religion und Politik, das in diesem Themenschwerpunkt im Mittelpunkt steht.

\subsection{Der Begriff dang und Konfuzianismus als Religion}

Ein solches Beispiel, das vormoderne und außerwestliche Aspekte vereint, liefert der Fall des vormodernen kaiserlichen China, dessen Struktur der öffentlichen Meinungsbildung und Politik seit der Han-Zeit (206 v. Chr.-220 n. Chr.) durch den Konfuzianismus (oder die $R u$ 儒-Tradition) als dessen Leitideologie bestimmt wurde. Konkret diente er in Debatten als Argumentationsgrundlage für politische Entscheidungen und als Prüfungsstoff für die Rekrutierung der Beamten und prägte damit eine neue gesellschaftliche und politische Elite bis zum Beginn der Republikzeit (1912-1949).

Der Fall des konfuzianisch geprägten chinesischen Systems bietet sich in doppelter Weise als Untersuchungsfeld zur Erprobung an: Zum einen finden wir hier einen chinesischen, also emischen, Begriff, der in der Forschungsliteratur üblicherweise mit Parteiung oder Faktion (engl. ,faction“) übersetzt wird und auf die bürokratische Binnenstruktur des kaiserlichen China und dessen Meinungs- und Gruppenbildungsstrukturen im generellen hinweist. Insofern kann hier auch ein wissenschaftlicher Keyterm in einer emischen, nicht westlichen Begriffsgeschichte einen Ansatzpunkt finden und genealogisch - über die üblicherweise zitierte westliche Genealogie hinaus - in Richtung der chinesischen Semantik erweitert werden.

Auf der anderen Seite bietet der chinesische, speziell der konfuzianische Fall auch besondere Herausforderungen, nämlich in bezug auf die viel diskutierte Frage (vgl. Chen 2013; Meyer 2014 u.a.) der Anwendbarkeit des Religionsbegriffs auf den chinesischen Konfuzianismus und damit der Unterscheidung von Religion und Politik überhaupt. Da die Unterscheidung in der bekannten Form durch Konstellationen der westlich modernen Geschichte geprägt wurde, die sich von außerwestlichen unterscheiden, kann die Frage nach dieser Unterscheidung nur mit einem bewusst heuristischen Ansatz angegangen werden, der sich der Herkunft dieser Unterscheidung hermeneutisch bewusst ist, jedoch deren Nutzen erproben will. Gerade wegen dieser Differenz zu westlichen Wissensordnungen bietet sich der Fall für eine Erprobung an, um zu zeigen, dass und inwieweit der Parteiungsbegriff auch in einem solchen Kontext analytisch gewinnbringend zur Identifikation spezifischer Problemstellungen im Feld von Religion und Politik angewandt werden kann. Konkret soll gezeigt werden, dass bzw. inwiefern die Frage nach Parteiungen (bzw. Bildung solcher Parteiungen) als Prisma für die Frage des Verhältnisses von Politik von Religion im chinesisch-konfuzianischen Kontext dienen kann. 


\subsection{Zur Frage des religiösen Charakters bzw. religiöser Elemente des Konfuzianismus}

Während also die Frage eines emischen Parteiungsbegriffs unproblematischer erscheint, bedarf die Frage nach dem religiösen Charakter des oder religiösen Elementen im Konfuzianismus weiterer Vorklärung. Zur grundlegenden Problematik des Konfuzianismus als Religion kann der Artikel auf Beobachtungen aus der extensiven Debatte hierzu zurückgreifen. Er wählt hierbei einen in der Forschungsdebatte begründeten Zweischritt bezüglich des Konfuzianismus als ganzem und bezüglich spezifischer, als religiös identifizierter Elemente.

Die Frage nach dem religiösen Charakter des Konfuzianismus gründet sich in der historischen Wahrnehmung von (Familien)Ähnlichkeiten zwischen der konfuzianischen Lehrtradition (chines. jiao 教) als ganzer und anderen Lehrtraditionen: So wurde in der wirkungsgeschichtlich bedeutsamen interkulturellen Begegnung der Jesuiten mit den verschiedenen chinesischen Traditionen der Begriff der secta nicht nur auf die eigene christliche Tradition, sondern auch die konfuzianische oder $R u$ Tradition angewandt (secta - oder lex-literatorum) (Standaert 1999, S. 121), somit wurde strukturelle Ähnlichkeit wahrgenommen. Zugleich entzündete sich hier der Ritenstreit um die Einordnung der Konfuzius- und Ahnenriten als ,zivil“ oder ,religiös“, die selbst Rückwirkungen auf den westlichen Diskurs zu Riten- und Religionsbegriff hatte (Standaert 1999, S. 128). Max Müller bezog in Zusammenarbeit mit dem Missionarssinologen James Legge (1815-1897) die konfuzianischen Klassiker in sein Projekt der Sacred Books of the East und somit in den beginnenden Weltreligionendiskurs mit ein. ${ }^{1}$ Das angeblich komplette „Fehlen“ eines (vormodernen) Religionsbegriff nach westlichem Muster führte Wilfred Cantwell Smith hingegen zu seiner bekannten Kritik am Religionsbegriff überhaupt - und seinem Vorschlag zur Aufgabe desselben (Smith 1962). Jedoch könnte die konfuzianische Tradition sehr wohl als kumulative Tradition im Sinne von W. C. Smith gelten, so zeigt sie auch funktionale Ähnlichkeiten zu anderen (religiösen) Traditionen. Ansätze wie der von Kleine haben darüber hinaus über den Ansatz von Familienähnlichkeit in Anlehnung an Wittgenstein - jenseits eines essentialistischen Verständnis von Religion - versucht, über strukturelle und semantische Ähnlichkeiten (im Aufweis von außerwestlichen Gattungsbegriffen) komparative Fragestellungen zu Religion wieder möglich zu machen. ${ }^{2}$

Mit Blick auf das chinesische Vokabular finden wir Gattungsbegriffe wie den Begriff jiao (Lehre, Lehrtradition), die verschiedene Lehrtraditionen umfassen können und sich strukturell (über Familienähnlichkeit) mit dem westlichen Religionsbegriff vergleichen lassen. ${ }^{3}$ Dies erweist sich auch an dessen Gebrauch in der chinesischen Geschichte: Der jiao-Begriff wurde zunächst im chinesischen Mittelalter zunächst für die drei Traditionen (,Drei Lehren“, sanjiao 三教) von Konfuzianismus,

\footnotetext{
1 Sun (2008); vgl. a. die Kritik am Weltreligionendiskurs bei Masuzawa (2005).

2 Kleine (2013), kritisch hierzu Bergunder (2011) mit einem Ansatz, der stattdessen die Frage nach Religion aus radikal diskursiv-genealogischer Sicht stellt, vgl. ähnlich Meyer (2020).

3 Zum Aufweis solcher vergleichbarer Gattungsbegriff, aber auch mit Hinweis insbesondere auf unterschiedliche Metaphoriken der verwandten Begrifflichkeiten s. Campany (2003).
} 
Buddhismus und Daoismus ${ }^{4}$ genutzt, aber in der Kulturbegegnung mit Islam und Christentum auch auf diese ausgeweitet. Um 1900 wurde sodann durch Kang Youwei 康有爲 (1858-1927) eine wirkungsgeschichtlich starke Tradition begründet, die konfuzianische Tradition mit dem Neologimus zongjiao 宗教 (als Übersetzungsbegriff für „Religion“) in Analogie zum Christentum zu verstehen, wobei insbesondere funktionale Aspekte im Sinne einer Volks- und Staatsreligion im Kontext von Stateund Nation-building im Vordergrund standen (Chen 1999; Meyer 2014). Andere chinesische Verfasser haben hingegen die Anwendung des zongjiao- oder Religionsbegriffs auf den Konfuzianismus wegen dessen angeblich rein säkularen Charakters als ethischer und gesellschaftlich-politischer Philosophie kritisiert - wobei sie einen westlichen Aufklärungsdiskurs zum Konfuzianismus seit Christian Wolff rezipierten. In neuerer Zeit haben Vertreter des philosophisch orientierten modernen Neukonfuzianismus wie Liu Shu-hsien und Tu Wei-ming jedoch eine religiöse Dimension oder religiöse Aspekte eingeräumt. ${ }^{5}$

In der sinologischen Forschung hat sich ein Trend herausgebildet, über die konfuzianische Tradition als ganze und deren funktionale Aspekte hinaus konkreter auf Elemente zu fokussieren, die im Vergleich leichter als religiös in einem engeren Sinne zu klassifizieren sind, so etwa die in der textlichen und rituellen Tradition vorfindlichen Vorstellungen eines Himmels (tian 天) sowie von Geistern (shen 神), inkl. Natur-, Orts- und Ahnengeistern, sowie entsprechende Opferrituale, die sich an solche Entitäten richten. Die stärkere oder geringe Bedeutung solcher Aspekte in der Gesamtlehre auch bei einzelnen (neo)konfuzianischen Vertretern (z. B. Zhu Xi 朱喜, 1130-1200) (Ching 2000; Tillman 2019) wird zunehmend thematisiert. Relativ unstrittig ist darum die Identifikation der Rolle von übermenschlichen Entitäten wie Himmel und verschiedenen Geistern inkl. Ahnengeistern und entsprechenden (Opfer)riten als religiös. Ebenso aber auch, dass gesellschaftlich-politische Aspekte ebenso zum konfuzianischen Erbe der $R u$-Tradition gehören, welche den Begriff des Religiösen im interkulturellen Vergleich sprengen. ${ }^{6}$ Es hat sich somit ein Forschungskonsens entwickelt, einerseits Konfuzianismus, d. h. die konfuzianische Tradition als Ganze, in die Frage nach Religion einzubeziehen - und zugleich dessen Charakter als zugleich gesellschaftsethische und politische Lehre zu problematisieren -, andererseits auf spezifische Aspekte, die in einem engeren Sinne als religiös einzuordnen sind, zu fokussieren.

\subsection{Vorgehen und Thesen}

Entsprechend wird sich dieser Artikel - nach dem ersten Abschnitt zur Geschichte des Terminus dang - dem damit bezeichneten Phänomen von Parteiungsbildungen

\footnotetext{
${ }^{4}$ Zum Drei-Lehren bzw. sanjiao-Diskurs und zur westlichen Rezeption des Drei-Lehren (sanjiao)-Diskurses im Westen u. a. Gentz (2006).

${ }^{5}$ Bei Liu Shu-hsien mit Rückgriff auf Tillichs Konzept eines ,ultimate concern“ mit Betonung der Wertedimension (Ethik) im Konfuzianismus (vgl. a. die Rede einer „,immanenten Transzendenz“); bei Tu vorwiegend mit Bezug auf die Selbstkultivierungstradition, vgl. Meyer (2014, S. 205-206).

${ }^{6}$ Deren Verbindungen - u. a. die legitimierende Rolle des Himmels für die dynastische Herrschaft - können in diesem Zusammenhang nicht in weiterem Rahmen untersucht und diskutiert werden, s. hierzu aber unsere Diskussion unten zum Adoptionsfall.
} 
im Spannungsfeld von Religion und Politik in zwei Stufen annähern. In einer ersten Stufe wird die Frage der Rolle ideologischer Ausprägungen der konfuzianischer Lehrtradition - anhand einflussreicher ideologischer Bewegungen - als Faktor neben anderen Faktoren (u. a. Mechanismen des Verwaltungsapparates und externer politischer Herausforderungen) anhand des Ablaufs der Parteiungsstreitigkeiten des 11. Jh. untersucht. Dabei wird vorausgesetzt, dass konfuzianische Tradition an sich trotz seiner Instrumentalisierung als Staatsideologie nicht mit dem Staat in eins zu setzen ist, sondern eine bleibende Differenz zwischen dieser Tradition und dessen Rolle im Staat besteht. Der Abschnitt wird dabei zunächst die Struktur der ,hofzentrierten Öffentlichkeit“ (Meyer 2008), sodann die konkreten Parteiungs- oder Faktionsstreitigkeiten im Kontext der ideologischen Bewegungen von Guwen 古文 (,Altstil“), Wang Anshis Xinxue 新學 (,Neuer Lehre“) und beginnender (neokonfuzianischer) Daoxue 道學 (,Lehre des Weges“) diskutieren.

In einem zweiten Schritt wird auf religiöse Opferriten am Hof als typisches religionspolitisches Thema anhand der Ritendebatten des 11. Jh. als Fallbeispiele fokussiert. Dabei greift der Verfasser auf von ihm untersuchtes Material zurück, das hier zusammenfassend im Fragekontext vorgestellt werden soll. Diese Debatten können von anderen Feldern politischer Diskussionen (Finanzen, Militär, aber auch Beamtenrekrutierung) unterschieden werden. Der Gegenstand der kaiserlichen Opferriten und Opfermusik hatte hohen symbolischen Wert. Untersucht werden soll darum die Relevanz solcher Ritenstreitigkeiten für Parteiungsbildungen in der hofzentrierten Öffentlichkeit und innerhalb der Verwaltungsbürokratie.

Insgesamt vertritt der Artikel zwei Hauptthesen:

1. Zum einen argumentiert er für die Anwendbarkeit eines Keyterms Parteiung über den westlich-modernen Kontext hinaus auf vormodern chinesische Kontexte, hier den konfuzianischen Kontext, wobei der chinesische emische Begriff dang 黨 und seine Genealogie als Anknüpfungspunkt dienen. Der Begriff verweist insbesondere auf Konstellationen und Mechanismen der Meinungs- und Gruppenbildung innerhalb einer Verwaltungsbürokratie sowie die negative Wertung solcher Gruppenbildungen als Spezifika der vormodernen chinesischen Genealogie des Begriffs.

2. Zum anderen erweist sich der Begriff als produktiv, um das Verhältnis von Religion und Politik in Meinungs- und Gruppenbildungsprozessen zu reflektieren. Hierzu argumentiert der Artikel, dass der Fokus auf - oft fluide - Bildungen von Parteiungen (dang) hilft, den Stellenwert bestimmter gesellschaftlicher und/oder diskursiver Konfliktlinien für die politische Gruppenbildung zu erkennen. Er greift hierbei auf die oben skizzierte operationelle Unterscheidung in zwei Stufen zurück. Während es phasenweise eine deutliche Korrelation bzw. Überschneidung von politischen Gruppenbildungen (sog. „Reform-“ und „konservative“ Gruppen) mit der Guwen-Bewegung, Wang Anshis „Neuer Lehre“ (Хіпxиe) und der neokonfuzianischen Daoxue-Bewegung im 11. Jh. gibt, stellten Ritenfragen zwar eine hochsymbolische Materie dar, die jedoch seltener und nur für kürzere Dauer gruppenbildende Wirkung hatte (etwa im Fall einer Adoption des Kaisers). 
Zugleich ist gegenüber ideologischen Richtungsfragen (Guwen, Xinxue, Daохиe) und Ritenfragen jedoch auch stets der institutionelle Rahmen in Form der bürokratischen Ämter und deren Mechanismen der politischen Meinungs- und Gruppenbildungen zu beachten, insbesondere da Netzwerke oder Seilschaften notwendige Voraussetzung für Förderung von Karrieren innerhalb der Verwaltungsbürokratie darstellten. Politische Gruppenbildungen im Spannungsfeld von Religion und Politik können darum nur in einem komplexen und dynamischen Ineinanderwirken dieser Aspekte verstanden werden.

\subsection{Zum Forschungsstand}

Der Artikel kann auf bestehende Forschung zur Erforschung des Begriffs dang im vormodernen China und zu Faktions- (Pengdang朋黨-)Streitigkeiten im 11. Jh. zurückgreifen. Die Anwendung der Übersetzung Parteiung (van Ess 2003, S. 36 und 169; Höckelmann 2016) oder faction (Wechsler 1973; Daby 1979; Hartwell 1986; Kurz 1998; Strange 2008; Levine 2008) ist dabei etabliert. Die Anwendung und Begründung des Begriffs erfolgten jedoch größtenteils kontextgebunden historisch. Wechsler (1973, S. 87) hat den Begriff allgemein definiert als ,, a subgroup in a decision-making body working for the advancement of certain policies or people“. Dalby (1979, S. 639-654) hat ebenfalls für die frühe Tang-Dynastie (7. Jh.) die eher lockere Verbindung dieser Netzwerke betont. ${ }^{7}$ Höckelmann (2016, S. 176f) bezieht sich in seiner Definition auf den Politikwissenschaftler Köllner (2004, S. 3), der Faktion als ,informelles Beziehungssystem“ bestimmt; eine Faktion sei ,jede relativ organisierte Gruppe, die im Kontext einer anderen Gruppe existiert und welche (als eine politische Faktion) mit ihrem Rivalen im Wettstreit um Machtvorteile innerhalb der größeren Gruppe steht, von der sie ein Teil ist." Charakteristisch ist danach die schwache Institutionalisierung und Fluidität solcher Bildungen von Untergruppen innerhalb größerer meinungs- und entscheidungsbildender Gruppen (hier besonders innerhalb des sozialen Kontinuums von konfuzianischen Gebildeten und daraus rekrutierter Beamtenschaft, institutionell mit Fokus innerhalb der Bürokratie), wobei diese Untergruppen durch gemeinsame inhaltliche undloder personenbezogene Motivationen und Interessen konstituiert werden.

Zudem gibt es ausführliche westliche und chinesische Forschung zu den wechselnden Bildungen von Parteiungen im Kontext der Song-Zeit, besonders des 11. Jh., im Zusammenhang verschiedener Reformversuche (Fan Zhongyan 范仲淹 (989-1052), Wang Anshi 王安石); dabei sind auch die neuen ideologischen Formierungen des Konfuzianismus von der sogenannten Altstil- (Guwen古文-)Bewegung über die Neue-Lehre des Reformers Wang Anshi bis hin zur Formierung der Gruppe und Denkrichtung des sogenannten Neokonfuzianismus (Daoxue 道學, Lixue 理學) intensiv untersucht worden, auch bezüglich ihrer Verbindungen zu bzw. ihrer Wirkung auf die Formierung von Parteiungen (u. a. Luo Jiaxiang 1993; Vittinghoff 1975; Meyer 2008, 2011; de Weerdt et al. 2020). Nicht explizit thematisiert wurde die Fragestellung bisher im Spannungsfeld von Religion und Politik. Meine

\footnotetext{
7 Dalby (1979, S. 639): ,... loose associations among politicians, arising from complex networks of personal relations that defy easy characterization.“
} 
eigenen Forschungen (Meyer 2008, 2011), die die rituellen Elemente als Kristallisationspunkte politisch-ideologischer Meinungsbildung herausgearbeitet haben, können als Ausgangspunkte und Grundlage für die hier dargelegten Reflexionen und Schlussfolgerungen dienen.

Im ersten Schritt wird nun der vormoderne emische Begriff dang und dessen Prägung als diskursiver Begriff vorgestellt, bevor wir auf die Anwendung zur Frage von Religion und Politik kommen.

\section{Kontext und Entstehung des Begriffs, dang '(Parteiung) im vormodernen chinesischen Diskurs bis zum 11. Jh.}

\subsection{Herkunftskontext}

Der locus classicus für den Begriff dang im späteren Sinne findet sich in einem kurzen Abschnitt der „Gespräche des Konfuzius“ (des Buchs Lunyu 論語), Kap. XV, S. 22:

Der Meister (Kong) sprach: „,Der Edle ist selbstbewusst, aber nicht streitsüchtig, er liebt den Umgang mit Menschen, aber betreibt keine Gruppenbildung [zum eigenen Nutzen] (oder: ist nicht parteiisch) [dang]. "8

Das hier verwandte chinesische Lexem dang war keineswegs von Anfang an negativ konnotiert. Es bedeutete zunächst eine lokal basierte Gruppe, teilweise auch eine Verwandtschaftsgruppe (Clan), aber dann auch abstrakter andere nicht auf lokale Herkunft, Familie oder Clan beruhende Gruppenbildungen (Luo Zhufeng 1994, S. 1364 f). Laut dem zhanguo- oder hanzeitlichen Klassiker Zhouli ,handelte es sich bei dang im Altertum um einen Personenverband oder eine Verwaltungseinheit von 500 Familien“9 Erst später nahm der Begriff die Konnotation von „Parteilichkeit“ im Sinne egoistischer Interesse und fehlender Orientierung am Gemeinwohl an (Luo Zhufeng 1994, S. 1365, vgl. Höckelmann 2016, S. 178; zu weiteren Verwendungen in konfuzianischen Klassikern u. a. im Shangshu vgl. a. Levine 2005, S. 159-163,).

Die Textstelle im Lunyu ist zunächst vor dem Hintergrund der Zeit der Periode der ausgehenden Chunqiu-Zeit (ca. 722-479 v. Chr.) und der Streitenden Reiche (Zhanguo-Periode, 478-221 v. Chr.) in der chinesischen Antike zu sehen. Wir befinden uns hier in einer Phase der Transformation von der aristokratischen Gesellschaft der Zhou-Dynastie zu neuen zentralistisch regierten Einzelstaaten, in denen auch Nichtadlige wichtige Positionen als Berater oder später Beamte erringen konnten. In diesem Kontext ist das Ideal des Edlen (junzi 君子) zu verorten, das auf Konfuzius (Meister Kong 孔, 549-479 v. Chr.) selbst zurückgehen könnte (z. B. Bauer 2001, S. 58 f). Dieser bildete vermutlich als Lehrer Anwärter aus klein- und nichtadli-

\footnotetext{
8 《論語·衛靈公》子曰:「君子䂆而不爭「, 群而不黨。」 Lunyu XV, S. 22, s. https://ctext.org/ analects/wei-ling-gong (chinesisches Original und englische Übersetzung, letztere lautet dort: The Master said, ,The superior man is dignified, but does not wrangle. He is sociable, but not a partisan.“); Übersetzung hier weitgehend angelehnt an Moritz (1998, S. 102 (Nr. 22)), Übersetzung von dang abweichend.

9 Höckelmann (2016, S. 178) mit Bezug auf den Klassiker Zhouli, vgl. a. Levine (2005, S. 156).
} 
gen Familie für die konkurrierenden Fürstenhöfe aus. Das genannte Ideal des Edlen (junzi, wörtlich „Sohn des Fürsten“) verkörperte dabei ein Tugendbild der stets im Geflecht der hierarchischen Beziehungen korrekt und für das Allgemeinwohl (gong 公) handelnden sowie nie auf das Eigenwohl bedachten Person.

Dieses Ideal wurde später von der konfuzianisch gebildeten Elite der Beamten im chinesischen Kaiserreich ab der Han-Zeit (ab ca. 2. Jh. v. Chr.) übernommen. Die politische Realität war jedoch schon in der Chunqiu- und Zhanguo-Periode mit ihren sich bekämpfenden Feudalstaaten eine andere. Berater an den Höfen aus verschiedenen Denkschulen konkurrierten miteinander, vertraten ganz andere Konzepte als die Konfuzianer und rangen um Einfluss für sich und ihre Ideen. So gab es die utilitaristisch ausgerichteten „Legalisten“ (fajia 法家), die eine strenge Herrschaft durch Gesetze vertraten, aber auch andere weltanschaulich geprägte Gruppen wie Daoisten (daojia 道家) und Kosmologische Schule (yinyangjia 陰陽家). ${ }^{10}$

Das Zitat und die Verwendung von dang an dieser Stelle kann somit im Kontext genau dieser Hofsituation mit verschiedenen Beratern verortet werden, die den Herrscher und damit die Meinungsbildung zu beeinflussen trachteten, ohne dass das Zeichen dang hier schon einen festen Begriff oder damit gar eine formale „Partei(ung)“ bezeichnet hätte. Der - allerdings erst etwas später a posteriori in der HanZeit - für diese Gruppen bzw. deren Denkrichtungen geprägte Sammelbegriff war jia 家 (Schulrichtung) - nicht dang. Er bezeichnete eher formal zusammenfassend die Denkrichtung, teilweise auch eine auf einen Gründer (Konfuzius, Mozi) zurückgehende Schule, nicht so sehr eine konkrete Gruppenbildung an einem spezifischen Fürstenhof. Jedoch ist das bekannte Stichwort der „Meister und Hundert Schulen“ (zhuzi baijia 諸子百家) oder „Hundert Schulen, die miteinander wetteifern“ (baijia zhengming 百家爭鳴) bezeichnend, um das Phänomen pluraler Meinungsbildung im damaligen politischen Kontext festzumachen.

Der Begriff dang gewann erst in der Kaiserzeit ab der Han-Dynastie (207 v. Chr.-220 n.Chr.) eine neue Bedeutung. Als fester Begriff kennzeichnet er mit vorwiegend negativer Konnotation „Seilschaft“ oder „Netzwerk“ in der Bürokratie, die egoistische Interessen verfolgten. ${ }^{11}$ Mit der chinesischen Kaiserzeit haben wir eine komplett neue Situation, die das Verhältnis von Staat und Weltanschauung (inkl. religiös konnotierter Elemente wie Ahnenverehrung, Himmels- und anderer Opfer, Ritenwesen etc., Moral) prägte. Konkret kam es im Laufe des 2. Jh. in der frühen Han-Dynastie zur allmählichen Durchsetzung des Konfuzianismus als einer Art Staatsorthodoxie. So führte Kaiser Wu 武 (reg. 141-87 v. Chr.) der Han-Dynastie die Fünf kanonischen Klassiker (wujing 五經) aus der konfuzianischen „,Ru 儒“-Tradition als Bildungsstoff der neuen Beamtenelite ein (Einrichtung einer kaiserlichen Universität 124 v. Chr.). Diese fungierten als normative Argumentationsgrundlage in Diskussionen (Vogelsang 2013, 157-163). Bestimmte Elemente in den Klassikern dienten der Legitimation der monarchischen und dynastischen Herrschaft, die als vom Himmel eingesetzt galt (tianming 天命), solange sich die Herrscher moralisch verhielten. Kritik an der Regierung konnte in mehr oder weniger direkter Weise

\footnotetext{
10 Zu Überblicken zu diesen Schulen s. z. B. Bauer (2001, S. 151-116); Graham (1989); Schleichert und Roetz (2009).

11 Höckelmann (2016, S. 178) mit Bezug auf Liu Xiang 劉向 (77-6 v. Chr.) aus der Han-Dynastie.
} 
durch die konfuzianisch gebildeten Beamten persönlich in mündlich vorgetragenen Throneingaben geübt werden. Die Beamten erlangten hierdurch Einfluss auf die Politik. Auch das über mehrere Jahrhunderte kompilierte Lunyu (,Gespräche des Konfuzius") gewann neben den Fünf Klassikern normative Bedeutung, und so auch der obengenannte locus classicus - und damit das Verdikt der Bildung von Parteiungen. Dieses wurde nun auf die Gruppenbildungen von Beamten am Hof angewandt. Erst in dieser Konstellation mit einem fest etablierten Beamtenstaat samt seinen Institutionen und dessen Verknüpfung mit der konfuzianischen Ideologie - samt dessen religiös-rituellen Elementen - haben wir somit die Situation, in welcher der dang-Begriff seine eigentliche Karriere erlebte.

Erstmals wurde er auf den Machtkampf zwischen zwei Gruppen im Staat in der Späteren oder Östlichen Han-Dynastie (24-220 n. Chr.) explizit angewandt (Höckelmann 2016, S. 178). Später finden wir den Begriff insbesondere in der Tang-Dynastie (618-907 n. Chr.) in Anwendung auf eine weitere Machtkonstellation zweier Gruppen am Hof (u. a. Wechsler 1973, S. 87; Dalby 1979, S. 639-654). In diesen Debatten wurde der dang-Begriff mit dem genannten Lunyu-Ausspruch zitiert, um die jeweils andere Seite zu kritisieren. Auch die Präzedenzfälle aus der Han-Dynastie wurden in historischer Argumentation unter diesem Stichwort herangezogen und interpretiert. Neben Ideologie spielten oft Karriereinteressen und Seilschaften eine Rolle. In der Tang-Dynastie standen diese noch in Verbindung mit familiären und Heiratsbanden, da die Tang-Bürokratie noch stark durch aristokratische Familien beherrscht wurde. In seiner Analyse zum 9. Jh. hat Höckelmann ${ }^{12}$ hier einen wichtigen Unterschied zur folgenden Song-Dynastie ausgemacht, bei der er eine weitere Entwicklung des Phänomens sieht. ${ }^{13}$ So erklärt er die Häufung von innerchinesischen Diskursen zu Parteiungen in der folgenden Song-Zeit durch die wachsende Institutionalisierung der Zivilverwaltungsbürokratie sowie durch die Ablösung von traditionellen Mustern politischen Verhaltens, während in der Tang-Dynastie noch aristokratische Familien die Ämter und die Hofpolitik dominierten.

Die Song-Dynastie bietet, wie wir sehen werden, in der Tat ein besonderes Beispiel, da hier eine komplett neue Elite auftrat, die sich ganz auf die zivile Grundlage des erneuerten konfuzianisch-basierten Bildungssystems stützte. Diese Situation ist kennzeichnend für die folgende Kaiserzeit bis zu ihrem Ende 1911 und bietet herausragende Beispiele von Debatten um und zwischen Parteiungen in der chinesischen Bürokratie.

\footnotetext{
12 Höckelmann (2016, S. 176-181) mit Schwerpunkt auf dem 9. Jh. und Li Deyus Schrift Pengdanlun; zum Faktionalismus in der frühen Tang-Zeit (7. Jh.) besonders Eisenberg (1999, 2002-03) sowie Wechsler (1973).

${ }^{13}$ Höckelmann (2016, S. 176f) nimmt den Punkt der ,Einbettung von Faktionen in ein Evolutionsmodell politischer Institutionen“ (bei Köllner als Vorläufer von Parteien im modernen Sinne) positiv auf und überträgt dies auf die chinesische vormoderne Entwicklung.
} 


\subsection{Der dang-Diskurs in der Nördlichen Songzeit (960-1126 n. Chr.): Streit um die Legitimität von Parteibildungen der Edlen und Ouyang Xius „Pengdanglun“"}

Einen Höhepunkt erreichten die Diskussionen um Parteiungen und den dang-Begriff im 11. Jh. Bereits oben war der Kontext des locus classicus aus dem Lunyu erläutert worden. Hier erscheint das Bild des Edlen, der nicht streitet und keine egoistische Gruppenbildung betreibt. Im Gegensatz dazu steht das negative Bild des auf seinen Vorteil bedachten Beraters oder Beamten. Diesem wird Gruppenbildung als Mittel der Durchsetzung seiner Partikularinteressen gegenüber dem Gemeinwohl unterstellt. Der Begriff dang oder pengdang (Freundesgruppen/-cliquen) war, wie wir gesehen hatten, in der Han- und Tang-Zeit im Kontext des kaiserlich-bürokratischen Staats als durch und durch pejorativer Begriff neugeprägt worden (Levine 2005, bes. 156). Faktisch stand der Begriff für das Verdikt jeglicher Gruppenbildung - die sich zugleich als unvermeidbar im bürokratischen System zeigte. „Spalter“, die eine dang bildeten und so das Ideal einer einheitlichen und uneigennützigen Beamtenschaft sprengten, waren in diesem Sinne stets die anderen. ${ }^{14}$

Den Widerspruch der Unvermeidbarkeit der Gruppenbildung und des Verdikts versuchte der wichtige Guwen- und Reformvertreter Ouyang Xiu 歐陽修 (1007-1072) in einem kontrovers diskutierten ${ }^{15}$ Debattenbeitrag „Zum Thema der Parteiungen von Freunden“ („Pengdanglun 朋黨論“) zugunsten der eigenen Gruppe um den Reformführer Fan Zhongyan logisch aufzuheben. Unmittelbarer Kontext war der Vorwurf gegenüber Fan Zhongyan und seinen Anhängern eine Parteiung oder Freundesgruppe zu bilden. Der Text soll hier zur Illustration als längeres Zitat wiedergegeben werden. Er argumentiert, wie folgt:

Euer Untertan hat gehört, daß es die [abschätzige] Redeweise von den Parteiungen und Freundesgruppen seit alters gibt. ... Im allgemeinen bilden Edle mit Edlen Freundesgruppen, weil sie denselben Weg verfolgen, und Nichtswürdige bilden Freundesgruppen mit Nichtswürdigen, weil sie nach demselben Vorteil streben. ... Doch Euer Untertan sagt, daß [in Wahrheit] nur die Edlen Freunde haben, die Nichtswürdigen aber nicht. Woran liegt das? Was die Nichtswürdigen begehren, das sind Gewinn und Gehalt, wonach sie gieren, das sind Güter und Reichtum. Daß sie sich, solange sie denselben Vorteil sehen für eine kurze Zeit als Parteiung zusammentun und eine Freundesgruppe bilden, ist nur trügerisch. Sehen sie einen Gewinn, dann streiten sie um den Vortritt, und wenn der Gewinn aufgezehrt ist und die Verbindung sich lockert, dann morden und

\footnotetext{
14 Lorge (2010, S. 313) fasst die Situation in der Song-Zeit in seiner Rezension zu Levine (2008) treffend zusammen: „Generally speaking, despite several attempts to change this, factions were not theoretically acceptable at court. A faction was, by definition, an evil cabal of unscrupulous officials bent on undermining the just power of the emperor. Good men would naturally be ideologically and morally unified and serve the emperor as part of a selfless bureaucracy. Since officials were selected for their moral attainment as much as for their intellectual capabilities, service in government required moral perfection. And since it was not ideologically possible for morally perfect men to disagree profoundly, when court officials did come into irreconcilable conflict, one side had to be not just wrong, but morally compromised."

$15 \mathrm{Zu}$ diametral entgegengesetzten und eher vermittelnden Positionen s. Levine (2005, S. 199f).
} 
versehren sie einander stattdessen. [...] Anders ist das bei den Edlen. Was diese bewahren, das sind der rechte Weg und die Rechtlichkeit ... Dienen sie damit dem Staat, dann haben sie dieselben Ziele und sorgen gemeinsam für Ausgewogenheit (van Ess 2004, S. 189).

Hauptargument war somit die Sozialität der „Edlen“ (junzi) vs. der Asozialität der egoistisch Handelnden, die überhaupt nur kurzzeitige Interessengemeinschaften bilden könnten. Auf persönlichen Nutzen ( $l i$ 利) angelegtes Denken war im Konfuzianismus seit der Antike verpönt und stand im Gegensatz zum Allgemeinwohl (gong 公). Gruppenbildung, die auf egoistischen Partikularinteressen ausgerichtet war, wurde im Gegensatz zum uneigennützigen Edlen gesehen. Das Argument bei Ouyang arbeitet weiterhin mit einer moralischen Unterscheidung von Edlen vs. unmoralisch Handelnden und bleibt höchst normativ aufgeladen.

Der Text ist bemerkenswert, weil er einerseits an der negativen Wertung von - nach Ouyang stets nur kurzzeitig angelegten - Gruppenbildungen der Nichtswürdigen (Gemeinen) festhält, und damit an einer moralischen Wertung von Politik. Andererseits versucht er aber den Begriff der Parteiung (pengdang) selbst - mit dem ja die Gruppierung um Fan Zhongyan bezeichnet wurde, zu der Ouyang Xiu gehörte (s. unten 3.2.) - aus dem klassischen semantischen Gefüge zu lösen und zu rehabilitieren: nicht nur die Unwürdigen, sondern auch - bzw. gerade - die Edlen bilden Freundesgruppen. Seine Theorie der Parteiungen ähnelt allerdings trotz der positiven Umwertung in keiner Weise einem modernen Begriff von Partei, da sie an einem moralischen Politikbegriff gebunden ist. Eines solches Politikverständnis ließ gerade keinen Parteien- und Meinungspluralismus zu, der mit legitimen Partikularinteressen und deren Widerstreit rechnet. Stattdessen markiert der Begriff einen moralisch-ideologischen Wahrheitsanspruch. Trotzdem bildete der Versuch Ouyangs eine Basis für die spätere Aufnahme bei Liang Qichao 梁啓超 (1873-1929) im frühen 20. Jh.

In seiner Zeit konnte sich Ouyangs Versuch der Umprägung des Begriffs nicht durchsetzen, blieb aber als Meinungsäußerung eines prominenten Intellektuellen weiter präsent. Er markiert für uns den dang-Begriff als semantischen Marker eines durchaus realen Phänomens. Gerade die Kollision von ideologisch-normativem Wahrheitsanspruch und unvermeidbaren Gruppenbildungsmechanismen wurde dabei am Begriff problematisiert und weist so zugleich auf ein grundsätzliches Problem eines vormodernen Politikverständnisses hin, das zur lokalen Genealogie des Begriffs in Ostasien gehört. ${ }^{16}$

\subsection{Ausblick: Die moderne Rezeption und Weiterwirkung in die Gegenwart}

Der Begriff dang hatte, wie wir gesehen haben seit der Han-Zeit einen festen Platz in der Selbstwahrnehmung politischen Handelns im vormodernen China. Selbst wenn er überwiegend negativ konnotiert war (und für die Bezeichnung eigener Gruppenbildung problematisch war), beschrieb er eine Realität innerbürokratischer Gruppenbildung und der kollektiven Meinungsbildung in der Beamtenschaft. Die Position,

16 Zur Kritik eines solchen Politikverständnisses in China noch des frühen 20. Jh. s. Fröhlich (2000). 
die Ouyang Xiu in seiner Abhandlung vorgebracht hatte, war zwar umstritten und wurde nie zur Mehrheitsposition, blieb jedoch durch die Prominenz Ouyangs präsent; die Debatten der Song-Zeit bildeten insgesamt einen Referenzrahmen in der Genealogie des Begriffs bis zum 20. Jh. ${ }^{17}$

Die Aufnahme dieses Begriffs als Übersetzung des westlichen Konzepts von Parteien war darum vorbereitet und wurde in der Krise des späten Kaiserreichs am Ende der Qing-Dynastie (1644-1911) durch den konfuzianisch-klassisch gebildeten Intellektuellen Liang Qichao geleistet. Dieser war zunächst als Parteigänger des konfuzianischen Reformers Kang Youwei während des - nur kurzzeitig erfolgreichen Reformversuchs von 1898 (der sogenannten 100-Tage-Reform) am Ende der QingDynastie aufgetreten. Die Mechanismen der Meinungsbildung in der Bürokratie und darum auch ähnlich von Gruppenbildungen funktionierten dabei nicht viel anders als im kaiserlichen China zuvor. Die Krise des Systems (angesichts der technischmilitärischen Überlegenheit des Westens) und der Einfluss westlicher Ideen nötigten Liang in seinem Exil in Japan nach 1898 zur verstärkten Übernahme westlicher Ideen. Seine Lektüre von Schriften zur politischen Theorie führten ihn dabei weg von einem ursprünglich von ihm unterstützten Modell einer konstitutionellen Monarchie (präferiert von seinem Mentor Kang Youwei) hin zur Befürwortung einer parlamentarischen Demokratie mit Parteien. In diesem Kontext nahm Liang - nicht zuletzt unter Einfluss von ihm gelesener japanischer Schriften (Fogel 2004 u.a.) den Begriff dang auf, als er nach vorhandenen Vorbildern und Begriffen in der chinesischen Geschichte suchte. Dabei wird sein Rückgriff auf Ouyang Xiu deutlich, wenn er den Begriff positiv anwendet. Er verbindet ihn hier mit seinem westlich geprägten Konzept des „,neuen Bürgers“ (xinmin 新民). In der Übernahme des Begriffs nach Ouyang ergab sich jedoch ein konzeptionelles Problem: einzig legitime Gruppenbildung konnte nach diesem Verständnis nur diejenige der moralisch Guten, der Edlen (junzi-dang 君子黨), sein; und die eigene Partei sollte selbstverständlich diese eigentlich legitime dang sein. Dies Verständnis schlägt sich auch bei Liang selbst nieder, obwohl er sich zugleich auf das moderne westliche MehrparteienModell bezieht. Die überkommene Semantik stand somit im Gegensatz zur Idee des Parteienparlaments als Modell eines pluralistischen Interessenausgleichs und eines Mehrparteiensystems. In seinen Schriften und seinem Handeln konnte Liang diesen Gegensatz nur schwer ausgleichen (vgl. Judge 1995).

Auch über Liangs Person hinaus und nach der Revolution und Etablierung der chinesischen Republik 1912 mit der Einführung eines Parlaments und Parteien wie der Guomindang (Nationalpartei) des Revolutionär Sun Yat-sens fehlte eine politische Kultur des Parteienpluralismus. ${ }^{18}$ Zunächst bildeten sich Parteiengruppen im Parlament der neuen Republik. In Verfassungsdiskussionen formierte sich eine konfuzianische Gruppe im Gegenüber zu Vertretern, die den Konfuzianismus eher

\footnotetext{
${ }_{17} \mathrm{Zu}$ weiteren Äußerungen in den folgenden Dynastien mit Bezug auf die Song-Debatten s. z. B. Wang Fuzhi 王夫之 (1619-1692) (Wang Fuzhi 1985, 10.201; De Weerdt et al. 2020, S. 348). Eine vollständige und umfassende Analyse späterer, insbesondere auch moderner adaptiver Interpretationen um $1900 \mathrm{kann}$ in diesem Rahmen nicht geleistet werden.

18 Vgl. hierzu der Artikel von Ellguth (2021) in diesem Themenschwerpunkt. Vgl. a. Fröhlich (2000) passim, mit explizitem Bezug auf den pengdang-Begriff besonders Fröhlich (2000, S. 40).
} 
zurückdrängen wollten. Jedoch gab es ansonsten keine explizit religiösen (buddhistischen oder christlichen) Parteien. Auch in der Folgezeit ging der Trend eher hin zur Dominanz von Einparteiensystemen - gepaart mit dem Verständnis von (nur) einer eigentlich legitimen Partei - und ideologischen Parteien: So bildete die Nationalpartei (Guomindang) mit dem Sunyatsenismus ihre eigene Ideologie aus, ebenso auch die 1921 gegründete Kommunistische Partei Chinas. Beide Parteien waren auf dem chinesischen Festland, bzw. die Nationalpartei ab 1949 bis zur Aufhebung des Kriegsrechts auf Taiwan, beherrschend. Kleinere Parteien wie die vom Intellektuellen Zhang Junmai mitgegründete sozialdemokratische Partei hatten es schwer und waren faktisch marginalisiert.

Die historischen Konnotationen mit ihrer starken negativen Wertung hängen der Idee von modernen dang oder Parteien im chinesischsprachigen Kontext in Form einer begriffsgeschichtlichen Last bis heute an. Sie sind zu lesen als wirksamer Teil der Genealogie der gegenwärtigen Einparteiendiktatur der Kommunistischen Partei Chinas, in welcher weltanschaulicher Pluralismus, Dissens und ideologische Devianz gerade keinen legitimen Raum haben und auch Religionen allenfalls toleriert und kontrolliert werden. Unter der neuen Ägide von Parteichef und Staatspräsident Xi Jinping erleben wir die kontinuierliche Einschränkung der in den Reformjahren seit 1978 gewährten Freiräume und eines tolerierten Dissenses hin zu einer neuen totalitären Gleichschaltung. Die Möglichkeit einer erfolgreichen - und über Liang Qichao hinausgehenden - Überschreibung der Begriffe im westlich-demokratischen Sinne bietet das aktuelle Taiwan (und in Abstufung Hongkong bis in jüngere Zeit). So hat Taiwan seit der Aufhebung des Kriegsrechts 1987 einen mehrmaligen Regierungswechsel zwischen den Parteien erlebt, wobei auch hier das Parteienmodell von Demokratie teilweise kritisch diskutiert wird. Dessen Probleme ähneln jedoch eher denen westlicher Demokratien.

\subsection{Zusammenfassung}

Der dang-Begriff erscheint darum stets normativ aufgeladen. Begriffsgeschichtlich mit Fokus auf dem vormodernen China und der Song-Zeit ist er nicht zu trennen vom aus moderner Sicht problematischen Ineinander von Ideologie und Politik. Er wirft damit aber auch ein Schlaglicht auf den westlichen Parteienbegriff, der normativ einen Pluralismus und moderne Institutionen eines Mehrparteienparlaments als Ort der Meinungsbildung in Unterscheidung von der Exekutive voraussetzt. Die Anwendung des modernen Begriffs Partei passt darum nur schwer auf vormoderne und außerwestliche Konstellationen. Der Begriff der Parteiung hilft hier vorschnellen Übertragungen westlich-moderner Modelle für die Bildung allgemeiner analytischer Begriffe zu entgehen und sich auf andere Konstellationen im Feld von Politik und Weltanschauung einzulassen.

Festzuhalten aus diesem knappen Überblick zur semantischen Geschichte des Terminus dang bleibt somit einerseits die Existenz eines solchen emischen Begriffs im chinesischsprachigen ${ }^{19}$ Kontext, wobei dieser fast durchgehend negativ geprägt

19 Die historischen Semanitk des Begriffs dang in anderen ostasiatischen Kontexten der Sinosphere (Japan, Korea, Vietnam) kann hier nicht untersucht werden. 
war, und dessen Prägung eine Belastung für den heutigen dang-Begriff bzw. das Parteienverständnis darstellen mag. Der Begriff selbst entstammt der ideologischen Tradition der konfuzianischen Schriften. Der Fokus in der folgenden Analyse soll jedoch auf dessen Anwendung im Kontext der Song-Zeit im Spannungsfeld von Religion und Politik liegen.

\section{Analyse: Die Formation von Parteiungen in der Nördlichen Song- Dynastie im Spannungsfeld von Religion und Politik}

Die Situation in der Song-Dynastie stellt für uns in mehrfacher Hinsicht ein interessantes Fallbeispiel dar: Erstens finden wir hier eine auffällige Häufung an wechselnden Konstellationen von Parteiungen in kurzer Zeit. Zudem ist zweitens für uns relevant, dass diese nicht nur mit wichtigen politischen Reformbewegungen des 11. Jh. (Fan Zhongyan, Wang Anshi), sondern zeitlich auch mit einem ideologischen Revival des Konfuzianismus koinzidierten, das auf der erhöhten Bedeutung und dem erneuerten Selbstverständnis der Elite der Zivilbeamten beruhte. Drittens finden wir hier eine Reihe von kontinuierlichen Debatten zu kaiserlichen Opferriten und deren Ritenmusik, deren Verhältnis zur Formierung von Parteiungen untersucht werden kann. Eine wichtige Voraussetzung für die offenen Auseinandersetzungen bildete schließlich die besonders starke Ausprägung einer offenen Diskussionskultur im 11. Jh. am Hof, d. h. innerhalb der zentralen Beamtenschaft, und darüber hinaus.

Das ideologische Revival des Konfuzianismus beinhaltete allgemein eine bewusste Rückwendung zu den antiken Klassikern in Stil und nach den dort beschriebenen Vorbildern ( fugu 復古). Am Anfang und in der Mitte des 11. Jh. formierte sich diese Richtung zunächst in Gestalt der Altstil- oder Guwen-Bewegung. Aus dieser entstammte auch die Neue Lehre (Хіпxue) des Reformers Wang Anshis. Im späteren 11. Jh. etablierte sich die spezielle Richtung des Neokonfuzianismus (Daoxue, Lixue).

Das Verhältnis von ideologischen Prägungen und konkreter politischer Gruppenbildung wird nun genauer zu untersuchen sein. In einem ersten Abschnitt sollen in kurzer Analyse die institutionellen Strukturen der Bürokratie der Nördlichen SongDynastie und ihrer Mechanismen der Meinungs- und Entscheidungsbildung (3.1.) untersucht werden, in dem die Gruppenbildungen stattfanden. Im zweiten Schritt sollen dann anhand eines Überblicks des Ablaufs der Formierungen und Neuformierungen der Parteiungen im 11. Jh. die Faktoren, die zu deren Bildungen führten, analysiert werden im Hinblick auf interne Mechanismen, externe politische Faktoren und ideologische Bewegungen (besonders der Guwen-Bewegung und der neokonfuzianischen Daoxue) (3.2.). Mit dem Fokus auf Riten soll das Verhältnis von Religion und Politik im Zusammenhang mit der Bildung wechselnder Parteiungen (dang) dann noch einmal am Fallbeispiel mehrerer Ritendebatten über mehrere Dekaden hinweg verfolgt werden (3.3.). 


\subsection{Kontext: Struktur der Institutionen, hofzentrierte Öffentlichkeit und Ablauf der Streitigkeiten}

Ein Charakteristikum der Song-Dynastie ist die Blüte des bürokratischen Beamtenstaats und des Konfuzianismus - seiner ideologischen Grundlage. Die Strukturen von Ämtern und Abläufen stellen die institutionellen Voraussetzungen für die diversen offen ausgetragenen Streitigkeiten und sich daraus ergebnenden Meinungs- und Gruppenbildungsprozesse dar.

Unterschiedliche Institutionen und Mechanismen, die wir bereits in der Han- oder der Sui- und Tang-Dynastie kennen, finden sich nach der militärischen Konsolidierungsphase in besonderer Vollendung ab der Zeit des Kaisers Renzong 仁宗 (reg. 1022-1063). Im Übergang von Tang- zur Song-Zeit entstand eine neue nichtadlige, auf klassischer Bildung beruhende Elite (shidafu 士大夫), der die zivile Verwaltung anvertraut wurde.

Kennzeichnend für die Entfaltung einer reichen Diskussionskultur waren dabei eine Reihe von Institutionen, die eine relativ freie und vielfältige Äußerung von Meinungen erlaubten und zugleich Strukturen pluraler Meinungsbildung vorgaben:

Zunächst zu den wichtigsten Ämtern: In Übernahme der Institutionen der TangDynastie fand sich ein Staatsrat mit verschiedenen Ministerien und ein ausgefeiltes System an Rängen, aber auch ad-hoc-Kommissionen, die u.a. zu Ritenfragen herangezogen wurden. ${ }^{20}$ Jedoch war diese Hierarchie nicht als reine Befehlshierarchie zu verstehen. Vielmehr konnten Beamte am Hof relativ frei Vorschläge über sogenannte -verschriftlichte und direkt vorgetragene - Throneingaben vorbringen. Zusätzlich fand sich noch ein besonderes Amt: Neben der Regierung (dem Staatsrat), der zusammen mit dem Kaiser die Exekutive darstellte, gab es das Amt der Zensoren, einer Institution, die es schon als Beamtenposition seit der Han-Dynastie gab und die Aufgabe hatte, Kritik an der Verwaltung bis hinauf zur Regierung (und indirekt somit am Kaiser) zu üben. Dieses wichtige Instrument bot eine institutionelle Möglichkeit zu Gegenmeinungen und somit zu Opposition zur Meinungsbildung der Regierung. Das Prestige dieser Zensorenämter und die damit verbundene Möglichkeit der Profilierung war so groß, dass ein solches Amt quasi ein Sprungbrett für spätere Regierungsämter darstellen konnte (Jia 1996), so dass eine innere Dynamik ermöglicht wurde, die einem Wechsel von Regierung und Oppositions(sparteien) in parlamentarischen Systemen ähnelt. Das Recht zum Vortrag eigener Vorschläge und das Zensoratsamt liefen als Instrumente somit einer rein hierarchischen Struktur der Verwaltungshierarchie zuwider und stellten die Grundlage für die Möglichkeit relativ freier Meinungsbildung über argumentativ ausgetragene Hofdebatten dar. Der Kaiser hatte dabei die wichtige Rolle als Letztentscheider und konnte sich als Schiedsrichter die verschiedenen Meinungen und Vorschläge anhören, bevor er eine Entscheidung traf. Zudem konnte er aktiv zur Sammlung von Meinungen (jiyi 集議) aufrufen; eine der häufigsten Fragen waren dabei nicht im modernen Sinne politische Fragen, sondern Klärungen zur angemessenen Ausführung von Riten, die als

\footnotetext{
$20 \mathrm{Zu}$ einer zusammenfassenden Darstellung der Institutionen der nördlichen Song-Dynastie mit Fokus auf die Diskussionskultur s. Meyer (2008).
} 
höchst relevant für die Staatsführung angesehen wurden (Ji 1990; Wu 1996). Dies wird in 3.3. noch näher ausgeführt werden.

Wir finden entsprechend auf dieser institutionellen Basis in der Song-Dynastie eine ausgesprochen offene Diskussionskultur. Über den Bereich des Hofs hinaus konnten auch nichtverbeamtete Angehörige der Elite an der Meinungsbildung inoffiziell partizipieren. Sogar auf eine ,,allgemeine Meinung“ (gonglun 公論; gongyi 公議) unter der Elite bzw. im Volk ${ }^{21}$ konnte in Argumentationen am Hof Bezug genommen werden. Wir können dabei (im Unterschied zu einem demokratischen Konzept, aber auch der Idee einer reinen Befehlshierarchie) von einem Modell hofzentrierter meinungsbildender Öffentlichkeit sprechen, die auf den Hof als Entscheidungszentrum fokussiert war, aber konzentrisch auch weitere Kreise (u. a. an privatern Akademien, shuyuan 書院) einbezog..$^{22}$ Grundlage der Argumentation waren die konfuzianischen Klassiker, aus denen moralische Vorbilder und Handlungsnormen extrahiert wurden. Neben einer Amtshierarchie, die Karrierechancen bot, finden wir also Freiräume für inhaltliche Diskussionen und argumentativen Austausch. Zugleich musste Meinungsbildung innerhalb der Beamtenschaft organisiert werden. Da formale Parteien nicht im System vorgesehen waren, erfüllten Regierungsvorschläge, ergänzt und vorbereitet durch Vorschläge aus der Verwaltung, adhoc-Kommissionen und freie Redebeiträge (Throneingaben) - sowie als Korrektiv Kritiken und Remonstrationen durch die Zensoren - die Funktion, eine argumentativ begründete Meinungsbildung dem Kaiser zur Entscheidung vorzutragen. Die Bildung von Parteiungen (dang) war, wie wir gesehen haben, offiziell gerade nicht vorgesehen und stand unter Verdikt, allerdings liefen Einreichungen von Vorschlägen und Gegenvorschlägen faktisch im Hintergrund koordiniert durch Netzwerke ab. Damit verbunden war, dass formal zwar anhand der offiziellen Institutionen, aber tatsächlich gerade innerhalb dieser Netzwerke sich Nachwuchsbeamte durch Throneingaben zum Zwecke ihrer Karriere profilieren konnten. Empfehlungen innerhalb der Bürokratie waren normalerweise der Weg für Beförderungen, wofür die Zugehörigkeit zu einem Netzwerk praktisch unabdingbar war.

Erst diese komplexen institutionellen Strukturen im kaiserlich-bürokratischen Staat stellten somit die Möglichkeit von freien Diskussionen, aber auch die Grund-

\footnotetext{
${ }^{21} \mathrm{Zu}$ den Begriffen gongyi und gonglun s. auch Maedje (2002, S. 11f). Ob diese stets eine Meinung im Volk allgemein oder eher innerhalb der Beamtenschaft bedeuteten, bleibt ambivalent. Gerade im unten genannten Adoptionsstreit (Puyi) findet sich dieser Begriff besonders häufig (Zhao 1999, S. 961, 969, 970 und 975). In einer Throneingabe (Zhao 1999, S. 964) wird der Ausdruck „öffentliche Meinung des ganzen Reiches" (tianxia zhi gongyi 天下之公議) benutzt, während der Gebrauch in einer anderen Throneingabe (Zhao 1999, S. 972-73) explizit auf die ,öffentliche Meinung unter den Hofbeamten” (juchao gongyi 舉朝公議) Bezug nimmt. In einer weiteren Eingabe (Zhao 1999, S. 977) wird der Begriff auf die öffentliche Meinung unter der Schicht der Gelehrten (shidafu), aus denen sich auch die Beamten rekrutierten, die jedoch über diese hinausging, bezogen (shidafu gongyi 士大夫公議). Das Volk ,auf den Straßen und Gassen" konnte jedoch zitiert werden, um die Meinung auch des normalen Volks zu repräsentieren. Im Allgemeinen wurde sicherlich die lokale und nationale Elite der shidafu als die repräsentativste Meinung außerhalb des Hofs angesehen, da sie die ,,interessierte und informierte Öffentlichkeit” über den Hof hinaus darstellte. Jedoch konnten insbesondere die Zensoren auf Stimmungen im Volk Bezug nehmen.

${ }^{22}$ Meyer (2008) in Anlehnung und Abgrenzung von Habermas entworfenen Begriff von Öffentlichkeit (Habermas 1969 [1962]), vgl. Meyer (2011).
} 
lage der Bildungen von Parteiungen (dang) dar, die im Folgenden analysiert werden sollen.

\subsection{Ablauf, Faktoren und Dynamiken der Bildung von Parteiungen im 11. Jh. und deren Verhältnis zu den ideologischen Bewegungen innerhalb des Konfuzianismus}

Im Laufe des 11. Jh. finden sich spätestens seit den 1030er Jahren eine Abfolge von Konstellationen und Streitigkeiten, die als Parteiungsstreitigkeiten (engl. „faction struggles“, chines. dangyi 黨議) in der Fachliteratur bekannt sind und die sich in der Südlichen Song-Zeit (1127-1279 n. Chr.) fortsetzten. Dabei handelte es sich, wie wir bereits oben skizziert haben, um informelle und inoffizielle Netzwerke vorwiegend von Beamten innerhalb der Verwaltungsbürokratie (und teilweise darüber hinaus innerhalb der Elite der Gebildeten ohne offizielles Amt), die sich entlang der offiziellen Strukturen der Öffentlichkeit bildeten und diese ergänzten. Sie dienten der Meinungs- und Entscheidungsbildung, aber auch der Förderung von Karrierewegen durch Empfehlungen.

In diesem Abschnitt soll die Verbindung von ideologischen Bewegungen innerhalb des Konfuzianismus und Bildung von Parteiungen in der nördlichen Song-Dynastie, also im 11. Jh., deutlich gemacht und analysiert werden. Zugleich wird hier ein Überblick über wechselnde Konstellationen von Parteiungsbildungen geboten, der den Blick differenzieren hilft. Eine Hauptthese ist, dass sich einerseits eine hohe Korrelation von ideologischen Bewegungen mit den Parteiungsbildungen bei wichtigen mittelfristigen Parteiungsbildungen zeigen lässt (die mittelfristigere Konfliktlinien abbildeten). Andererseits soll erkennbar werden, dass die Parteiungsbildungen nur aus einem komplexen Ineinander unterschiedlicher Faktoren zu verstehen sind.

Die Parteiungsstreitigkeiten, um die es hier geht, beginnen in den 1030er Jahren mit den ersten Gegensätzen zwischen einer jungen Generation von Reformen um ihren Führer Fan Zhongyan (Fischer 1955; Buriks 1956/57) gegen die Regierung unter dem langjährigen Kanzler Lü Yijian 呂夷簡 (996-1066). Die Reformer konnten in den 1040ern zwischenzeitlich und ab den 1050ern (mit der Führungsgestalt Ouyang Xiu) dann nochmals dauerhaft die Regierungsämter besetzen. Gegenstand der von Fan propagierten Reformen umfassten konkrete Probleme wie die Organisation des Militärs, Finanzen, aber auch Rekrutierung der Beamten und Schulwesen (Buriks 1956/57). Fan hatte zunächst Erfahrungen in den Provinzen gesammelt und kannte die lokalen Probleme vor Ort besser als viele Hauptstadtbeamte. Seine Reformideen führte er auf Vorbilder aus der Antike zurück. Besonders an seinen Anhängern wird die Verbindung zur Guwen-Bewegung explizit. So gilt Ouyang Xiu als einer der hervorragenden Vertreter der Guwen-Bewegung der Songzeit. Besonders deutlich wird die Verbindung von Politik und Ideologie in der Beförderung gleichgesinnter GuwenAnhänger auf Machtpositionen zuerst in den 1040ern und dann in den 1050-60ern. Im Examensjahr 1057 förderte Ouyang Xiu als Prüfungsverantwortlicher bewusst Anhänger der Guwen-Bewegung. Die Korrelation ideologischer Prägung mit der Reformgruppe um und nach Fan Zhongyan ist in dieser Phase darum besonders deutlich. 
Mit der Durchsetzung dieser Gruppe bildet sich jedoch in der folgenden Generation eine neue Gruppe Unzufriedener, die nun unter dem bekannten konservativen Politiker und Historiker Sima Guang 司馬光 (1019-1086) die Zensorenämter besetzten. Viele der Kritiker kamen aus Kreisen, die vorher als etabliert gegolten hatten, d.h. Familien, die bereits vorher Beamte hervorgebracht hatten und die oft nahe der Hauptstadt angesiedelt waren. Für diese Vertreter verbanden sich mit der Kritik insbesondere auch die Chance auf Karriere. Der Frust war besonders groß, da in den 1060er Jahren, die Regierungsämter des Staatsrats bereits mehrere Jahre lang von den gleichen Vertretern besetzt waren, so dass ein Beförderungsstau bestand. Die Kritik an den ehemaligen Reformern entzündete sich dann an einer Ritenfrage des aus einer Seitenlinie adoptierten Kaisers Yingzong 英宗 (reg. 1063-1067 n. Chr.) zur Frage der Verehrung seines leiblichen Vaters, des Fürsten von Pu, in der sogenannten $P u(y i)$ 誉議-Debatte. Dieser Fall rückte somit eine religiös-rituelle Frage in den Vordergrund, zu der wir unten noch einmal kommen werden. Die Debatte hatte zugleich politische Implikationen, da sie die Ordnung der Ahnenverehrung als symbolische Grundlage der Herrschaftslegitimation betraf. Der GuwenStil und die Ideen der Guwen-Bewegung hatten sich allerdings in dieser Zeit bereits durchgesetzt.

Ein erneuter Wechsel fand unter dem neuen Kaiser Shenzong 神宗 (reg. 1067-1085) statt, der den Reformer der nächsten Generation, Wang Anshi 王安石 (1021-1086), in die Regierung holte. Wangs Reformen ähnelten in verschiedenen Punkten den Reformvorschlägen Fan Zhongyans, tatsächlich war Wang auch anfänglich von Ouyang Xiu gefördert worden. Das galt allerdings auch für die SuFamilie, die sich nun gegen Wang stellt. Gegen Wang formierte sich darum unter dem konservativen, aus einer alten Familie kommenden Sima Guang (zu Sima Guang allgemein Ji 1998) eine vereinte Oppositionsgruppe sehr unterschiedlicher Provenienz, die nach dem Tode Shenzongs ab 1085 die Regierungsämter übernahm. Wir finden somit in dieser Zeit eine Ausdifferenzierung verschiedener Positionen nebeneinander. Neben Guwen-Ideen in Nachfolge Ouyang Xius und der speziellen „Neuen Lehre“ Wangs finden sich außer den konfuzianisch-konservativen Positionen Sima Guangs auch die sich formierende Daoxue-Lehre, die neokonfuzianische Schule unter Leitung der Brüder Cheng 程, welche ethische, ritologische und kosmologische Aspekte in neuartiger Weise kombinierte. Die Cheng-Brüder verband mit Sima Guang eine Herkunft aus einer alteingesessenen Beamtenfamilie, während Reformer oft eher homines novi waren. Sobald diese Opposition 1085 an der Regierung kam, zerfiel sie jedoch in konkurrierende Untergruppen. Ab 1093 führte die Übernahme der Regierungsgeschäfte durch den bis dahin unmündigen Kaiser Zhezongs 哲宗 (1077-1100, reg. 1085-1100) erneut zu einem Machtwechsel zugunsten der Anhänger des Wang Anshi und zu einer Unterdrückung ihrer Gegner durch Exilierung und Proskriptionslisten. ${ }^{23}$ Auch im 12. Jahrhundert setzten sich Machtkämpfe entlang der Meinungs- und Gruppenformierungsprozesse fort. ${ }^{24}$

\footnotetext{
23 Vittinghoff (1975), vgl. de Weerdt et al. (2020).

24 Vgl. de Weerdt (et al. 2020) u.a. mit einer computergestützten Auswertung der Netzwerke und Proskriptionslisten.
} 
Einen praktischen Hintergrund für die kontinuierlichen Reformversuche des 11. Jh. stellte die Finanzknappheit dar. Sie resultierte aus der militärischen Bedrohung im Norden durch die Liao遼-Dynastie der Khitan und die Xixia西夏-Dynastie der Tanguten, die zu teuren Friedensschlüssen (faktischen Tributzahlungen) und der Finanzierung eines riesigen Heeres schon in den 1030er und 1040er Jahren führten. Diese Belastung setzten sich bis zum Ende der Nördlichen Song-Zeit (1126) fort und stellte somit ein Dauerthema dar. Ebenso haben wir bereits gesehen, dass auch Zuordnungen zu ideologischen Richtungen eine Rolle spielten. Dabei ging es stets um konkurrierende Interpretationen der konfuzianischen Klassiker. Argumentiert wurde mit unterschiedlichen hermeneutischen Interpretationen der Klassiker und Argumentationsmustern des moralisch legitimierten Handelns. Die Reformer der 1030 er bis 1050er Jahre waren z. B. alle Anhänger der Guwen-Bewegung. Sie verbanden die Abwendung von einem formalistischen Schreibstil (pianwen 駢文) mit einer Orientierung nicht nur am Wortlaut einzelner Klassikerstellen, sondern dem Geist bzw. den Intentionen der weisen Herrscher der Antike und des Konfuzius zur Lösung von Problemen in der Gegenwart. Die neokonfuzianische Bewegung baute ebenso wie die Lehre Wangs auf der Guwen-Bewegung auf, verband die Interpretation der Klassiker jedoch mit einer rigideren und normativ strengeren Lesart, u. a. in Ritenfragen (s. unten), während Anhänger der Su-Familie wie Ouyang Xiu deutlich flexiblere, an die Gegenwart angepasstere Normen vertraten (Meyer 2008, 2011).

Allein der zeitliche Überblick lässt zudem den Wechsel von Generationen als ein weiteres Element in den Dynamiken der Gruppenbildungen erkennen. Dabei spielten Karrierechancen und -netzwerke eine Rolle. Personen in der Regierung versuchten ihre Anhänger zu fördern. Allerdings führte die langjährige Besetzung der höheren Ämter zu einem Beförderungsstau auf den unteren Ebenen. Auslöser konnten neben den praktischen Reformfragen auch symbolische Fragen der kaiserlichen Riten darstellen. Das Ineinander dieser unterschiedlichen Elemente - unterschiedliche politische Reformagenden, Karrieremechanismen in einer Beamtenhierarchie und ideologische Aspekte - soll mit Fokus auf den rituellen Fragen hinsichtlich der Bildung von Parteiungen (factions) unten weiter analysiert werden.

Es lässt sich somit festhalten, dass sich die meisten, besonders die längerfristigen Parteibildungen der 1030-60er-Jahre (Reformparteiung um Fan Zhongyan, Ouyang Xiu vs. Konservative zunächst unter Lü Yijian) sowie die zwischen dem Reformer Wang Anshi und seinen Gegnern (ab den späten 1060ern) stark mit ideologischen Bewegungen decken: Fan Zhongyan und seine Anhänger wie Ouyang Xiu kamen aus der Guwen-Bewegung (mit Ouyang Xiu als einem der bekannten Hauptvertreter). Dies heißt allerdings nicht, dass alle Guwen-Anhänger automatisch Anhänger des politischen Lagers von Fan waren. Wang Anshi, als Führer der Reform ab den 1060er Jahren, ist nicht ohne seinen frühen Förderer Ouyang Xiu zu verstehen, der sich jedoch in seinen späten Jahren von ihm distanzierte. Wangs Neue Lehre (Хinxue) wurde nicht zuletzt in Hinblick auf sein neues Bildungs- und Schulsystem durch den von ihm definierten Kanon geprägt. Die ideologischen Positionen seiner politischen Gegner waren jedoch keineswegs einheitlich: So waren die Mitglieder der Su-Familie Anhänger Ouyang Xius, die Gruppe Cheng Yis, aus dessen Schule sich dann die neokonfuzianische Daoxue-Tradition entwickelte, stand in scharfem Gegensatz zu den Sus - ein Gegensatz, der nach dem zwischenzeitlichen Sieg gegen 
die Wang-Anshi-Reformer 1085 aufbrach. Unterschiedliche Positionen in konkreten Fragen, aber auch der Kanonhermeunetik und nicht zuletzt Ritenfragen (s. unten) führten in der Periode 1085-1093 zu einer klaren Trennung zwischen diesen beiden Gruppen (neben einer weiteren Mittelgruppe). Der Pu(yi)-Streit Mitte der 1060er als weitere Parteistreitigkeit zwischen den beiden Reformperioden kann als Phänomen einer Übergangsperiode gesehen werden, in der sich Reformgegner formierten, wobei auch generationelle Gründe eine Rolle spielten. Auslöser war jedoch eine Ritenfrage, zu der wir darum noch kommen.

Der Überblick über die Debatten bestätigt somit zunächst die These einer hohen Korrelation von ideologischen Bewegungen mit den Parteiungsbildungen bei wichtigen mittelfristigen Streitfragen. Andererseits ist zu sehen, dass die Parteiungsbildungen nur aus einem komplexen Ineinander unterschiedlicher Faktoren zu verstehen sind. Es ist zu unterscheiden zwischen (1) systeminternen bzw. institutionellen Faktoren (Mechanismen der Verwaltungsbürokratie in der Meinungsbildung und Rolle von Netzwerken für Empfehlungen und Karrieren), (2) externen Faktoren politischer Herausforderungen (Reformbedarf) und (3) ideologischen Überzeugungen (die oft mit sozioökonomischen Interessen der Vertreter korrelierten). Die hochsymbolische Materie der kaiserlichen Opferriten (des Staatskults) kann als spezielles (religionspolitisches) Feld von Sachfragen angesehen werden, in dem sich Religion und Politik direkt überschneiden und dessen Rolle als Faktor für Parteiungsbildungen zu untersuchen ist.

\subsection{Religiös-rituelle Streitpunkte (Debatten um Hofriten)}

Der Fokus soll nun auf den rituellen Aspekten liegen, da sich hier am klarsten - neben ideologischen und hermeneutischen Aspekten der Klassikerinterpretation religiöse von eher praktisch-politischen Fragen differenzieren lassen. Zudem spielten Ritendebatten, wie bereits gesehen, eine auffallende Rolle als Auslöser einiger Streitigkeiten zwischen Parteiungen bzw. als Faktor in der Bildung derselben. Ihr Stellenwert muss jedoch genauer analysiert werden.

Im chinesischen Kontext stellte der Staatskult eine der Grundlagen des antiken Staates (noch vor Konfuzius) dar und wurde vom Konfuzianismus von seinen Anfängen an als Feld seiner Kernkompetenzen betrachtet. Die richtigen kaiserlichen Riten, und hierbei insbesondere die Ahnenriten und die kaiserlichen Opfer an den Himmel, stellten die legitimatorische Basis für die Herrschaft der Dynastie und der einzelnen Kaiser dar. Die Ritenmaterie spielte genau darum eine bedeutende Rolle in hofzentrierten Debatten. Nicht zuletzt bei einem Herrscherwechsel tauchte die Frage der Legitimation regelmäßig auf. Mit dieser Materie adressieren wir somit einen Bereich, der unmittelbar unsere Fragestellung von Religion und Politik und deren Bezug auf Parteiungsbildung betrifft. Auffallend ist hier zudem der starke Einbezug gebildeter Akteure außerhalb der Bürokratie, womit eine erweiterte - jedoch weiterhin hofzentrierte - Öffentlichkeit einbezogen wurde.

Die Untersuchungsmaterie von Debatten im Zeitraum von ca. 1034-1093 n. Chr. soll hier summarisch vorgestellt werden. ${ }^{25}$ Dabei lassen sich verschiedene Debat-

${ }^{25} \mathrm{Zu}$ einer genauen Analyse der relevanten Debatten im Zeitraum von ca. 1034-1093 s. Meyer (2008). 
tenfragen (kaiserliche Opferriten, die kaiserlichen Opferritenmusik yayue 雅樂, der Adoptionsfall des Yingzong-Kaisers Puyi und ein weiterer Einzelstreit) sowie Phasen (anhand der Regierungswechsel bzw. Änderungen der Parteiungskonstellationen) unterscheiden.

Zunächst ist festzustellen, dass die Ritenmaterie einen wichtigen abgrenzbaren Teil chinesischen normativen Wissens ausmachte. Dies zeigt sich in eigenen Kapiteln über Riten ( $l i$ 禮) in vormodernen Enzyklopädien und speziellen Ritenhandbüchern. ${ }^{26}$ Konkret geht es hier um besonders prominente Ritenfragen, die am Hof heftig diskutiert wurden, nämlich insbesondere die kaiserlichen Opferriten (jili 祭禮) gegenüber dem Himmel und den Ahnen sowie die diese begleitende Ritenmusik. Diese Opferriten gegenüber Himmel, Natur- und Ahnengeistern lassen sich im Kontext eines westlichen Religionsverständnisses via Familienähnlichkeit am klarsten als religiöse Frage identifizieren. Zudem stellten sie im chinesischen Kontext eine hochsymbolisch aufgeladene Materie dar. Die nur vom Kaiser vollziehbaren Himmelsopfer kennzeichneten den Herrscher als einzig legitimen Herrscher, der das Mandat des Himmels (tianming 天命), ${ }^{27}$ über das Reich zu herrschen, erhalten habe. Die Ahnenopfer legitimierten moralisch, insofern der Kaiser die oberste Tugend der Kindespietät gegenüber seinen Eltern und Vorfahren sichtbar praktizierte, aber auch indem er die Verbindung zum Gründerahn der Dynastie herstellte. Ritenmusik besaß bei allen Opfern einen hohen Stellenwert, da dieser die Herstellung eines kosmischen Bezuges und einer Harmonie zugeschrieben wurde.

Eine genaue Analyse der Debatten der mittleren Nordsong-Zeit (Meyer 2008, 2011) kann allerdings zeigen, dass der Stellenwert ritueller Fragen auch variieren konnte:

Eine erste Materie bieten die im Untersuchungszeitraum (1034-1093) auffallend kontinuierlichen Ritendebatten um die Ausgestaltung der Himmelsopfer und separate Debatten um die Ritenmusik bei kaiserlichen Ahnen- und Himmelsopfern. So wurden schon die Streitigkeiten um die Reformversuche in den 1030-40er-Jahren (mit der Reformer-Parteiung unter Fan Zhongyan) und dann diejenigen in den 1070-80er-Jahren (Reformgruppe unter Wang Anshi) von parallelen Ritendebatten begleitet. Diese Debatten zeigen tatsächlich eine erstaunliche Kontinuität über mehrere Jahrzehnte. Beitragende waren zum Teil prominente Gelehrte und aktive Beamte, zum Teil Experten in Ritualfragen, die separat hinzugezogen wurden. Diese Debattenteilnehmer lassen sich dabei meist auch einer der jeweiligen Parteiungen zuordnen. Mitglieder in eigens zusammengestellten Kommissionen wurden von dem einen oder anderen Netzwerk empfohlen. Teilweise spielten persönliche Verbindungen durch gemeinsame lokale Herkunft ${ }^{28}$ oder Familienbande durch Ver-

\footnotetext{
26 In Enzyklopädien wie dem Tongdian 通典 aus der Tang-Dynastie (618-907) umfassen die Kapitel zu $l i$ (Riten) allein einhundert der insgesamt zweihundert Kapitel (juan 卷) (vgl. Meyer 2011, S. 99).

$27 \mathrm{Zu}$ dieser auf den Wechsel von Shang- zu Zhou Dynastie im 11. Jh. v. Chr. zurückgeführten Ideologie s. z. B. Vogelsang (2013, S. 63 und 71; Opitz 1968, S. 26-34).

28 So waren die Parteiung um die Su-Brüder nach deren Herkunft aus Sichuan als Shu[=Sichuan]-dang 蜀黨 bekannt, die des Cheng Yi aus Luoyang als Luo[yang]-dang 洛黨. Eine weitere Gruppe war die mit Herkunft aus dem Norden verortete Shuo-dang 朔黨. Zur wechselnden Rolle von Hauptstadt-orientierten und eher lokal orientierten Netzwerken s. unten de Weerdt et al. (2020.
} 
heiratung eine Rolle. Teilweise war eher eine ideologische Nähe in der Auffassung der Klassiker relevant.

So war Hu Yuan 胡瑗 (993-1059) ein Experte für Ritenmusik, der nicht am Hof, sondern als Lehrer tätig war. Er wurde - ebenso wie zunächst die reformorintierten Beamten Feng Yuan 馮元 (975-1036) und Song Qi 宋祁 (998-1062) - auf Vorschlag des Reformführers Fan Zhongyan 1036 als Experte in eine Kommission für die Rekonstruktion der antiken Ritenmusik berufen und legte federführend einen Versuch der Rekonstruktion antiker Glocken in einer Schrift Huangyou xinyue tu $j i$ 皇祐新樂圖記 (,Abbildungen und Anmerkungen zur Neuen Musik der Huangyou-Periode“) vor (Meyer 2008, S. 92, 200-219). In den 1050er Jahren war er bei einem weiteren Anlauf zur Revision der Ritenmusik beteiligt und wurde später u. a. zum Lehrer an der kaiserlichen Universität Taixue befördert. Ein anderes Beispiel ist Fan Zhen 范鎮 (1008-1088), der schon damals mit Vorschlägen mitwirkte, vor allem aber nochmals in den 1070er und 80er-Jahren in einer neueren Debatte zu Ritenmusik in den Vordergrund trat. Er war ein Parteigänger ${ }^{29}$ des „Konservativen" Sima Guang, dessen Einfluss ihn in die damalige Kommission brachte. In einer ersten Phase um 1080 wurde die Kommission allerdings von Anhänger der Wang-Parteiung dominiert, Fan Zhen konnte immerhin aufgrund einer Empfehlung und Expertise teilnehmen. Nach dem Wechsel der Reformgegner in die Regierungsämter nach 1085/86 konnte er von Sima Guang protegiert in einem neuen Anlauf seine eigenen Vorstellungen zur Ritenmusik durchsetzen (Meyer 2008, S. 219-250). Auch hier wirkte sich somit der Regierungswechsel aus und die Materie der Riten bzw. Ritenmusik war bedeutend genug, dass sich dieser Machtwechsel hierin niederschlug. Allerdings wird man kaum sagen können, dass diese Ritenfragen selbst parteiungsbildend waren, jedoch wurden sie als hochsymbolisches Feld im Kontext der Darstellung von Herrschaftslegitimation beachtet.

Die Materie stellte zudem für die Beteiligten - wie wir an Hu Yuan und Fan Zhen als typischen Beispielen hierfür gesehen haben - auch Profilierungsmöglichkeiten für eine Karriere dar. Für Empfehlungen und Beförderungen waren dabei Mitglieder der eigenen Netzwerke in der Bürokratie (hier: Fan Zhongyan, Ouyang Xiu, Sima Guang) entscheidend. Die Beteiligung an diesen Debatten um die hochsymbolische Materie der Riten spielte somit stets auch eine Rolle in der Stärkung der eigenen Position - sowohl der eigenen Gruppe (Parteiung) als auch der Karriereaussichten des einzelnen..$^{30}$ Es lassen sich somit sehr verschiedene Motive und Faktoren ausmachen, die zur Parteiungsbildung beitragen.

Zwei besondere Fälle zeigen, wie zumindest teilweise oder zeitweise auch Fragen der Riten als Konflikte in den Vordergrund rücken, Konfliktlinien prägen und auf diese Weise gruppenbildend wirken konnten.

Besonders prominent war der sogenannte Puyi养議-Fall, die Debatte um den leiblichen Vater des Kaisers Yingzong 英宗 (reg. 1063-1067). Durch die Adoption dieses Kaisers durch seinen Vorgänger aus einer Seitenlinie des Herrscherhauses ereignete sich in den 1060er eine Störung, da ein Adoptionsfall grundsätzlich eine rituell ungeklärte Situation darstellte. Unstrittig war dabei die notwendige Verehrung

29 Als neuer Begriff wäre hier wohl der Ausdruck ,Parteiungsgänger“ einzuführen.

$30 \mathrm{Zu}$ den Debatten allgemein s. Meyer (2008). 
des Adoptivvaters und seine rituelle Anrede als Vater(ahn) durch den adoptierten Nachfolger. Jedoch empfand der neue Herrscher auch eine persönliche Bindung gegenüber seinem leiblichen Vater. Dieser Bindung wollte er durch ein Opfer und Anrede als Vater ebenfalls Genüge tun. Während Mitglieder des Staatsrates, unter anderem der obengenannte Ouyang Xiu, dieses Anliegen als rituell legitim unterstützten, opponierten insbesondere die Zensoren, darunter Sima Guang, mehrheitlich vehement gegen dieses Ansinnen als Infragestellung der legitimen Herrscherlinie.

Dieser berühmte Puyi-Fall ${ }^{31}$ hatte so hohe Bedeutung, dass sich der Zensor Sima Guang als einer der Führer der opponierenden Gruppe profilieren konnte und die Regierung mit ihren Anhängern in die Defensive gerieten. Zugleich ist offensichtlich, dass sich hier ein Streit der Generationen (jüngere Zensoren vs. ältere Regierungsmitglieder) um Karrieremöglichkeiten mit einer Frage verband, in der verschiedene Hermeneutiken der Schriftexegese der konfuzianischen Klassiker und Ordnungsmodelle kollidierten. Die damalige Regierung bestand zudem weitgehend aus Mitgliedern der früheren Reformgruppe um Ouyang Xiu, die vor allem ihre eigenen Anhänger gefördert hatten. Der Fall beleuchtet zudem die bleibende Differenz von auf den Kaiser fokussiertem Staat und Konfuzianismus als Ideologie, die keineswegs zu einem Kadavergehorsam führte. Die doppelte Rolle von Konfuzianismus als legitimierende Grundlage des chinesischen Kaisertums und als in den Klassikern hinterlegte (aber auf aktuelle Situationen hin zu interpretierende) Norm in den Händen der als Beamten fungierenden Gelehrten-Elite ermöglichte den Beamtengelehrten faktisch vom gegenüber dem Kaiser gebotenen Gehorsam abzuweichen und ihn an Normen zu messen, die durch sie selbst interpretiert wurden. Dies geschah in diesem Fall innerhalb der Beamtenschaft durch die Zensoren als wichtiger institutioneller Basis für die Bildung von devianten Meinungsgruppen innerhalb der Bürokratie. Der Puyi-Fall bietet damit ein besonders klares Beispiel für die Bedeutung ritueller Fragen abgesehen von eher realpolitischen Sachfragen einerseits, aber auch erneut für das Ineinander von politischer Gruppenbildung inklusiver handfester Karriereinteressen andererseits.

Ein dritter Fall am Ende der hier betrachteten Phase ist ebenfalls aufschlussreich: 1085 kam es durch den Tod Shenzongs, des Förderers der Wang-Anshi-Gruppe, zu einem Thronwechsel. Mit diesem kam das Netzwerk der Anti-Wang-Anshi-Parteiung (Anti-Reform-Gruppe) ${ }^{32}$ an die Macht. Dieses war allerdings vor allem durch den Gegensatz zur Wang Anshi zusammengehalten worden und in sich heterogen. Der Zerfall der Anti-Wang-Anshi-Koalition nach dem Thron- und Machtwechsel 1085 schließlich wird nicht zuletzt festgemacht am persönlichen Streit zwischen dem rigorosen Ritualisten Cheng Yi 程颐 (1033-1107) und Su Shi 蘇軾 (oder Su Dongpo東坡, 1037-1101), der eine flexible Anwendung in der Interpretation antiker Riten in der Gegenwart vertrat. Anlass waren hier die offiziellen Trauerriten um den verstorbenen Kanzler Sima Guang 1086. Der Ritualist und Führer der neokonfuzianischen Daoxue-Schulrichtung Cheng Yi war für die Riten als Verantwortlicher eingesetzt worden und vertrat auch hier eine strenge Orientierung an den antiken Riten. Su Shi, selbst in Regierungsverantwortung, fühlte sich an die strenge Auf-

31 Nach dem Namen des leiblichen Vaters; vgl. hierzu u. a. Meyer (2008).

$32 \mathrm{Zu}$ den Netzwerken mit geographischem Fokus s. a. die Arbeit von Freeman (1973). 
fassung nicht gebunden und machte sich über Chengs strengen Ritualismus lustig. Auch wenn tiefere Differenzen der Interpretation der konfuzianischen Tradition dem Zwist zugrunde lagen, war nicht zufällig eine Ritenfrage der Anlass für den offenen Hass zwischen beiden Persönlichkeiten und deren Anhängerschaften (Meyer 2008, S. 289-297). Auch hier lassen sich Ritenfragen und damit zusammenhängende Differenzen in den ideologischen Überzeugungen von persönlichen Netzwerken und Karriereinteressen - und in diesem Fall wohl auch persönliche Animositäten kaum voneinander trennen.

Generell waren Ritenfragen wie Ritenmusik und andere rituelle Detailfragen hoch prestigeträchtig und stellten ein wichtiges symbolische Kapital dar, das zugleich über Profilierung darin als soziales Kapital in der Karriere genutzt werden konnte. Wenn die Aufgabe in Kommissionen an Experten delegiert wurde, konnten dabei Experten der eigenen Gruppe bevorzugt wurden. Festzuhalten ist darum erneut das Ineinander von persönlichen Karriereinteressen, ideologischen Überzeugungen (nicht zuletzt an Ritenfragen und Interpretationen der Klassiker) und auslösenden tagespolitischen Sachfragen, die mit wechselndem Gewicht als Faktoren zu Gruppenbildungen beitragen konnten. Riten oder Ritenmusik konnten als Auslöser von kurzzeitigen Parteiungsbildungen fungieren und konnten in Parteiungskämpfen wichtige symbolisch aufgeladene Materien darstellen, eine bedeutende Frage wie der Adoptionsfall konnte als Ritenfrage tatsächlich eine deutliche Parteiungsbildung hervortreten lassen. Einzelfragen wie die Ritenmusik hatten aber kaum die Bedeutung und Wirkung, langzeitige Parteiungsbildungen zu prägen.

\section{Fazit}

Die Frage nach Parteiungen im konfuzianischen China führt mitten hinein in die Frage von Religion und Politik: Sie kann als Prisma für die Frage nach der Relevanz von „Religion“ bzw. religiösen Aspekten in Meinungs- und Gruppenbildungsprozessen dienen. Die Frage hat dabei heuristischen Charakter und bestätigt umgekehrt mit ihrer Anwendbarkeit den Nutzen der Fragestellung. Angesichts der vieldiskutierten Problematik des Konfuzianismus als religiös oder säkular (oder einer Verbindung beider Aspekte) kann eine solche Anwendung nur in vorsichtiger - und wie hier vorgeschlagen zweistufiger - Annäherung gewinnbringend geschehen.

Positiv festzuhalten ist dabei in der politischen Begriffsgeschichte Chinas zunächst die Existenz eines emischen Begriffs ,,dang“, der im vormodernen Kontext einer hofzentrierten räsonierenden Öffentlichkeit und entsprechenden Meinungsbildungsprozessen, als ungefähres Äquivalent zum Parteiungs- oder Faktionsbegriff angesehen werden kann und so in der Forschungsliteratur auch meist übersetzt wird. Es ist ein Begriff, der die Bildung von mehr oder weniger stabilen ,Parteiungen “ im Spannungsfeld von Weltanschauung und Politik als Gruppen- und Meinungsbildung entlang von wechselnden Konfliktlinien zu bezeichnen ermöglicht. In diesen Begriff ist die gesamte Genealogie der konfuzianischer Lehrtradition $(R u)$ aspektuell eingeschrieben: seine Geschichte als einzelne antike Beraterschule (jia) an den Fürstenhöfen der Zhanguo-Zeit in Konkurrenz zu anderen Schulen und Beratern, später seit der Han-Zeit aber die enge Verbindung mit dem kaiserlichen Beamten- 
system als dessen ideologische Grundlage. Trotz dieser Bindung konnten Vertreter der konfuzianischen Bildungselite jedoch in Distanz zum realen Staat in dessen Regierungshandeln treten und sich in ihrer Kritik auf den normativen konfuzianischen Kanon berufen. Zwischen Staat und Ideologie blieb somit eine Restspannung bestehen.

Die Übersetzung als Parteiung, ist gegenüber dem Parteibegriff zu bevorzugen, da es sich um Gruppenbildungen innerhalb einer institutionellen Einheit, nämlich einer Bürokratie, handelt, die informellen bis fluiden Charakter ohne formelle Mitgliedschaften hatten. Eine solche Binnendifferenzierung von Gruppen gerade innerhalb einer Bürokratie (auch in vergleichbaren modernen Kontexten) ist ein Phänomen, das wir durchaus auch anderswo finden und somit die Anwendung des Begriffs besonders nahelegt. Dabei offenbart die Begriffsgeschichte des dang-Begriffs, die wir hier mit Fokus auf der Song-Dynastie vorgestellt haben, nicht nur den bewussten diskursiven Charakter des Begriffs für entsprechende Phänomen der innerbürokratischen Gruppenbildung, der sich in eigenen Abhandlungen niederschlägt (u.a. im „Pengdanglun“ des Ouyang Xiu), sondern vor allem dessen pejorative Konnotation. Dieser politische Metadiskurs ist dabei selbst durch die Ideologie des Konfuzianismus als normativer Tradition und dessen ethisches Vorbild des Edlen (junzi) als unparteiisch Handelndem geprägt. Der Begriff dang ist hierbei an den normativen Begriff Dao 道 rückgebunden, dessen normativer Gültigkeitscharakter (als übermenschlich konnotierte Norm) für politisches wie individuelles Handeln stets vorausgesetzt wird.

In diesem semantischen und diskursiven Kontext ist auch die tatsächliche Bildung von Parteiungen im Kontext der Nördlichen Song-Zeit zu analysieren: Der Begriff erweist sich als hilfreich, um das Verhältnis von Religion und Politik in Meinungs- und Gruppenbildungsprozessen zu reflektieren. Der Artikel zeigt, dass der Fokus auf - oft fluide - Bildungen von Parteiungen (dang) hilft, den Stellenwert bestimmter gesellschaftlicher und/oder diskursiver Konfliktlinien für die politische Gruppenbildung zu erkennen. Dabei wurden hier in Hinblick auf die schwierige Frage des religiösen Charakters oder von religiösen Elementen im Konfuzianismus operationell zwei Schritte unterschieden:

Zum einen wurde die konfuzianische Tradition (als ganze, als jiao oder Lehre) in ihrer diskursiven Wirkung auf die Politik anhand konfuzianischer intellektueller Bewegungen des 11. Jh. untersucht. Das Verhältnis von Parteiungsbildungen und ideologischen Bewegungen zeigt in großen Phasen eine deutliche Korrelation bzw. Überschneidungen von politischen Gruppenbildungen (sog. ,Reform-“ und „,konservative" Gruppen, sowie Untergruppen) mit der Guwen-, Wang Anshis Neue-Lehreund der neokonfuzianischen Daouxe-Bewegung im 11. Jh., wobei sich diese mit weiteren Fragen sozialer Identität und generationellen Umformungen verbanden.

Die im zweiten Schritt untersuchten Ritenfragen stellten hingegen zwar eine hochsymbolische Materie dar, die jedoch seltener und nur für kürzere Dauer gruppenbildende Wirkung hatte (etwa in der Opposition zur Verehrung eines leiblichen Vaters des adoptierten Kaisers). Trotzdem wirkte die Materie der kaiserlichen Opferriten und Ritenmusik als attraktives Profilierungsfeld und konnte als Konflikt zeitweise auch Streitigkeiten und Gruppenbildungen auslösen.

Zusammenfassend lässt sich sagen: Grundlegend für Bildungen von Parteiungen waren neben karriereorientierten Seilschaften und Loyalitätsbindungen die Mecha- 
nismen chinesischer Bürokratie, die in Abwesenheit eines Parlaments eine wichtige Rolle als institutionalisierter Apparat der Meinungsbildung (und des Regierungshandelns) spielten (institutioneller Aspekt). Die Parteiungenbildungen verbanden sich aber über Herausforderungen in Sachfragen (Finanzen, Militär, Schul- und Examenswesen) hinaus regelmäßig mit ideologischen Ausrichtungen der Klassikerinterpretation und gaben diesen eine ideologische Grundierung. Parteiungsstreit entzündete sich zudem nicht selten an rituell-religiösen Fragen. Als solche spielten sie zwar nur im Ausnahmefall (Puyi-Fall) eine Rolle als eine Konfliktlinie, welche dauerhafte Bildungen von Parteiungen hervorbrachte, jedoch blieben sie in den Parteiungskämpfen durchgehend eine wichtige Arena und ein Profilierungsfeld.

Funding Open Access funding enabled and organized by Projekt DEAL.

Open Access Dieser Artikel wird unter der Creative Commons Namensnennung 4.0 International Lizenz veröffentlicht, welche die Nutzung, Vervielfältigung, Bearbeitung, Verbreitung und Wiedergabe in jeglichem Medium und Format erlaubt, sofern Sie den/die ursprünglichen Autor(en) und die Quelle ordnungsgemäß nennen, einen Link zur Creative Commons Lizenz beifügen und angeben, ob Änderungen vorgenommen wurden.

Die in diesem Artikel enthaltenen Bilder und sonstiges Drittmaterial unterliegen ebenfalls der genannten Creative Commons Lizenz, sofern sich aus der Abbildungslegende nichts anderes ergibt. Sofern das betreffende Material nicht unter der genannten Creative Commons Lizenz steht und die betreffende Handlung nicht nach gesetzlichen Vorschriften erlaubt ist, ist für die oben aufgeführten Weiterverwendungen des Materials die Einwilligung des jeweiligen Rechteinhabers einzuholen.

Weitere Details zur Lizenz entnehmen Sie bitte der Lizenzinformation auf http://creativecommons.org/ licenses/by/4.0/deed.de.

\section{Literatur}

Bauer, Wolfgang. 2001. Geschichte der chinesischen Philosophie. München: Beck.

Bergunder, Michael. 2011. Was ist Religion? Kulturwissenschaftliche Überlegungen zum Gegenstand der Religionswissenschaft. Zeitschrift für Religionswissenschaft 19:3-55.

Buriks, Peter. 1956. Fan Chung-yen's Versuch einer Reform des chinesischen Beamtenstaates in den Jahren 1043/44. Oriens Extremus 3:57-80 und Oriens Extremus 4:153-184.

Campany, Robert F. 2003. On the very idea of religions (in the modern West and in early medieval China). History of Religions 42(4):287-319.

Chen, Hsi-yuan. 1999. Confucianism encounters religion: The formation of religious discourse and the confucian movement in modern China. Ph.D. thesis. Harvard.

Chen, Yong. 2013. Confucianism as religion: Controversies and consequences. Leiden: Brill.

Ching, Julia. 2000. The religious thought of Chu Hsi. Oxford: Oxford University Press.

Dalby, Michael T. 1979. Court politics in late T'ang Times. In The Cambridge history of China, Bd. 3: Sui and T'ang China, 589-906 AD, part one. Hrsg. Denis Twitchett, 561-681. Cambridge: Cambridge University Press.

De Weerdt, Hilde, Brent Ho, Allon Wagner, Jiyan Qiao, und Mingkin Chu. 2020. Is there a faction in this list? Journal of Chinese History 4(2):347-389.

Eisenberg, Andrew. 1999. Installing an heir: Factional politics in the court of Tang Taizong. Tang yanjiu 5:247-268.

Eisenberg, Andrew. 2003. A study in court factionalism: The politics of Tang Taizong. Tang Studies 20-21:39-69.

Ellguth, Richard. 2021. Die Kuomintang und der Buddhismus in der Republik China (1912-1949): Zwischen Volkspartei und weltanschaulichen Parteiungen. Zeitschrift für Religion, Gesellschaft und Politik, online-Version ohne Seitenzahlen. https://doi.org/10.1007/s41682-021-00080-2. 
Ess, Hans van. 2004. Die Literatur der Dynastien Song und Yuan. In Chinesische Literaturgeschichte, Hrsg. Reinhard Emmerich, 187-224. Stuttgart, Weimar: Metzler.

Ess, Hans van. 2003. Von Ch'eng I zu Chu Hsi. Die Lehre vom Rechten Wege in der Überlieferung der Familie $\mathrm{Hu}$. Wiesbaden: Harrassowitz.

Fischer, Johanna. 1955. Fan Chung-yen (989-1052). Das Lebensbild eines chinesischen Staatsmannes. Oriens Extremus 2:39-85 und 142-156.

Fogel, Joshua A. (Hrsg.). 2004. The role of Japan in Liang Qichao's introduction of modern western civilization to China. Berkeley: Institute of East Asian Studies, Center for Chinese Studies, University of California.

Freeman, Michael Dennis. 1973. Lo-yang and the opposition to Wang An-shih: The rise of Confucian conservatism, 1068-1086. Ph.D. thesis. Yale.

Fröhlich, Thomas. 2000. Staatsdenken im China der Republikzeit (1912-1949). Die Instrumentalisierung philosophischer Ideen bei chinesischen Intellektuellen. Frankfurt, New York: Campus.

Gentz, Joachim. 2006. Die Drei Lehren (sanjiao) Chinas in Konflikt und Harmonie. Figuren und Strategien eine Debatte. In Religionen Nebeneinander. Modelle religiöser Vielfalt in Ost- und Südostasien, Hrsg. Edith Franke, Michael Pye, 17-40. Münster: LIT.

Graham, Angus Charles. 1989. Disputers of the Tao: Philosophical argument in ancient China. La Salle: Open Court.

Habermas, Jürgen. 1969. Strukturwandel der Öffentlichkeit. Untersuchungen zu einer Kategorie der bürgerlichen Gesellschaft, 4. Aufl., Neuwied, Berlin: Luchterhand. 1. Aufl. 1962.

Hartwell, Robert M. 1986. New approaches to the study of bureaucratic factionalism in Sung China: A hypothesis. The Bulletin of Sung-Yüan Studies 18:33-40.

Höckelmann, Michael. 2016. Li Deyu (787-850): Religion und Politik in der Tang-Zeit. Wiesbaden: Harrassowitz.

Ji Ping 季平. 1990. Song wangchao jiyi guoshi kaolun 宋王朝集議國事考論. Beijing Shifan daxue xuebao 北京師大學學報 4:32-37.

Ji, Xiao-bin. 1998. Conservatism and court politics in Northern Sung China: The thought and career of Ssu-ma Kuang (1019-1086). Ph.D. thesis. Princeton.

Jia Yuying 賈玉英. 1996. Songdai jiancha zhidu 宋代監察制度. Kaifeng: Henan daxue chubanshe.

Judge, Joan. 1995. The factional function of print. Liang Qichao, Shibao, and the fissures in the late Qing reform movement. Late Imperial China 16(1):120-140.

Kleine, Christoph. 2013. Religion als begriffliches Konzept und soziales System im vormodernen Japan: Polythetische Klassen, semantische und funktionale Äquivalente und strukturelle Analogien. In Religion in Asien? Studien zur Anwendbarkeit des Religionsbegriffs, Hrsg. Max Deeg, Oliver Freiberger, und Christoph Kleine, 225-292. Uppsala: Uppsala Universitet.

Köllner, Patrick. 2004. Faktionalismus in politischen Parteien: Charakteristika, Funktionen und Ursachen innerparteilicher Gruppen. Hamburg: Deutsches Übersee-Institut (DÜI-Arbeitspapiere 3/2004). http://epub.sub.uni-hamburg.de/epub/volltexte/2012/17279/. Zugegriffen: 20. Dez. 2020.

Kurz, Johannes L. 1998. The invention of a "faction" in Song historical writings on the Southern Tang. Journal of Song-Yuan Studies 28:1-35.

Levine, Ari Daniel. 2005. Faction theory and the political imagination of the Northern Song. Asia Major 18(2):155-200.

Levine, Ari Daniel. 2008. Divided by a common language: Factional conflict in late Northern Song China. Honolulu: University of Hawai'i Press.

Lorge, Peter. 2010. Rezension zu Ari Daniel Levine, Divided by a Common Language: Factional Conflict in Late Northern Song China. Honolulu, HI: University of Hawai'i Press, 2008. Zhongguo wenhua yanjiusuo xuebao 中國文化研究所學報/Journal of Chinese Studies 50:313-318.

Luo Jiaxiang 羅家祥. 1993. Beisong dangzheng yanjiu 北送黨争研究. Taibei: Wenjin chubanshe.

Luo Zhufeng 羅竹風 (Hrsg.). 1994. Hanyu dacidian 漢語大詞典, 2. Aufl., Shanghai: Hanyu dacidian chubanshe. 13 Bde.

Maedje, Stefan. 2002. Akademien, die Zhu-Schule und die Öffentlichkeit der Südlichen Song-Zeit (1127-1279): Eine Annäherung am Beispiel des Zhen Dexiu (1178-1235). Hamburg: Hamburger Sinologische Gesellschaft.

Masuzawa, Tomoko. 2005. The invention of world religions: Or, how European universalism was preserved in the language of pluralism. Chicago: University of Chicago Press.

Meyer, Christian. 2008. Ritendiskussionen am Hof der nördlichen Song-Dynastie 1034-1093. Zwischen Ritengelehrsamkeit, Machtkampf und intellektuellen Bewegungen. Sankt Augustin: Monumenta Serica. http://www.monumenta-serica.de/media/monumenta-serica/docs/Dokumente-publicationen/ Ritendiskussionen-Index-1-.pdf. Zugegriffen: 17.12.2021. 
Meyer, Christian. 2011. Negotiating rites in imperial China: The case of Northern Song court ritual debates from 1032 to 1093. In Negotiating Rites, Hrsg. Ute Hüsken und Frank Neubert, 99-115. Oxford, New York: Oxford University Press.

Meyer, Christian. 2014. Götter, Geister, der Weg und die Transzendenz im Konfuzianismus. In Gott und Götter in der Welt der Religionen, Hrsg. Markus Mühling, 178-218. Göttingen: Vandenhoeck \& Ruprecht.

Meyer, Christian. 2020. Zongjiao als chinesischer Religionsbegriff? Genealogische Anmerkungen zu seiner Entwicklung seit der späten Qing-Zeit. China heute 34(4):206-217.

Moritz, Ralf (Hrsg.). 1998. Konfuzius. Gespräche (Lun-yu). Stuttgart: Reclam.

Opitz, Peter. 1968. Chinesische Altertum und konfuzianische Klassik. München: List.

Schleichert, Hubert, und Heiner Roetz. 2009. Klassische chinesische Philosophie: Eine Einführung, 3. Aufl., Frankfurt: Klostermann.

Smith, Wilfred Cantwell. 1962. The meaning and end of religion: A new approach to the religious traditions of mankind. New York: Macmillan.

Standaert, Nicolas. 1999. The Jesuits did NOT manufacture ‘Confucianism’. East Asian Science, Technology, and Medicine 16:115-132.

Strange, Mark. 2008. Factionalism and the formation of eleventh-century military policy. Journal of SongYuan Studies 38:186-189.

Sun, Anna Xiao Dong. 2008. Confusions over Confucianism: Controversies over the religious nature of Confucianism, 1870-2007. Ph.D. thesis. Princeton.

Tillman, Hoyt. 2019. Zhu Xi's views on heaven, ghosts and spirits, and ritual. In Zhu Xi: Selected writings, Hrsg. Philip J. Ivanhoe, 116-137. Oxford: Oxford University Press.

Vittinghoff, Helmolt. 1975. Proskription und Intrige gegen Yüan-yu-Parteigänger. Ein Beitrag zu den Kontroversen nach den Reformen des Wang An-shih, dargestellt an den Biographien des Lu Tien (1042-1102) und des Ch'en Kuan (1057-1124). Bern, Frankfurt: Peter Lang.

Vogelsang, Kai. 2013. Geschichte Chinas, 4. Aufl., Stuttgart: Reclam.

Wang Fuzhi 王夫之. 1985. Song lun 宋論. Taibei: Liren shuju.

Wechsler, Howard J. 1973. Factionalism in early T'ang government. In Perspectives on the T'ang, Hrsg. Arthur F. Wright, Denis C. Twitchett, 87-120. New Haven: Yale Uinversity Press.

Wu Yining 吳以寧. 1996. Songdai chaosheng jiyi zhidu shulun 宋代朝省集議制度述論. Xueshu yuekan 學術月刊 10:56-62.

Zhao Ruyu 趙汝愚. 1999. Songchao zhuchen zouyi 宋朝諸臣奏議. Hrsg. Deng Guanglu 鄧廣銘 et al. 2 Bde. Shanghai: Shanghai guji chubanshe [urspgl.: Guochao mingchen zouyi 國朝名臣奏議]. 